hep-th/0408141

\title{
Stationary and Axisymmetric Solutions of Higher-Dimensional General Relativity
}

\author{
Troels Harmark \\ The Niels Bohr Institute \\ Blegdamsvej 17, 2100 Copenhagen Ø, Denmark \\ harmark@nbi.dk
}

\begin{abstract}
We study stationary and axisymmetric solutions of General Relativity, i.e. pure gravity, in four or higher dimensions. D-dimensional stationary and axisymmetric solutions are defined as having $D-2$ commuting Killing vector fields. We derive a canonical form of the metric for such solutions that effectively reduces the Einstein equations to a differential equation on an axisymmetric $D-2$ by $D-2$ matrix field living in three-dimensional flat space (apart from a subclass of solutions that instead reduce to a set of equations on a $D-2$ by $D-2$ matrix field living in two-dimensional flat space). This generalizes the Papapetrou form of the metric for stationary and axisymmetric solutions in four dimensions, and furthermore generalizes the work on Weyl solutions in four and higher dimensions. We analyze then the sources for the solutions, which are in the form of thin rods along a line in the three-dimensional flat space that the matrix field can be seen to live in. As examples of stationary and axisymmetric solutions, we study the five-dimensional rotating black hole and the rotating black ring, write the metrics in the canonical form and analyze the structure of the rods for each solution.
\end{abstract}




\section{Contents}

1 Introduction 1

2 Stationary and axisvmmetric solutions 4

2.1 Deriving canonical form of metric and the Einstein equations . . . . . . . . . . . . 4

2.2 Compact notation for the equations for $G_{i j}(r, z) \ldots \ldots \ldots \ldots$

3 Behavior of solutions near $r=0 \quad 9$

3.1 Behavior of $G(r . z)$ near $r=0 \ldots \ldots \ldots \ldots \ldots \ldots$

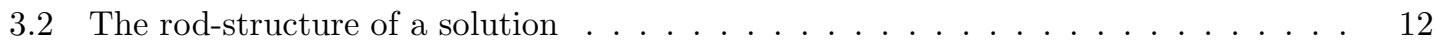

3.3 Analvsis of the rod-structure . . . . . . . . . . . . . . . . 13

4 Asvmptotically flat space-times

4.1 Perturbation of diagonal metrid . . . . . . . . . . . . . . . . . 15

4.2 Four-dimensional asvmptotic Minkowski-space $\ldots \ldots \ldots \ldots \ldots$

$4.3 \quad$ Five-dimensional asvmptotic Minkowski-space . . . . . . . . . . . . . . . . . 17

5 Rotating black hole solutions 18

5.1 Kerr solution . . . . . . . . . . . . . . . . . . . . . 18

5.2 Five-dimensional Mvers-Perrv solution . . . . . . . . . . . . . . . . . . . 20

5.3 Mvers-Perrv solution with one angular momentum . . . . . . . . . . . . . 23

6 Black ring solutions $\quad 24$

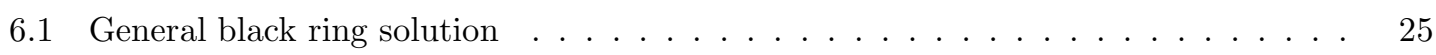

6.2 Regular rotating black ring $\ldots \ldots \ldots \ldots \ldots$

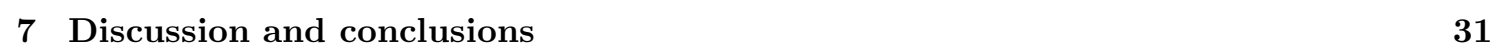

\begin{tabular}{|ll}
\hline A Special class of solutions & 32
\end{tabular}

B Analvsis of $\operatorname{det}\left(G_{i j}\right)$

\begin{tabular}{ll}
\hline C Diagonalizing a two-dimensional metrid & 34
\end{tabular}

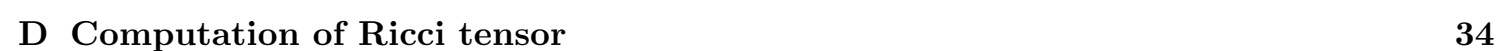

\begin{tabular}{|ll}
\hline E Properties of the equations for $G_{i j}(r, z)$ & 36
\end{tabular}

\begin{tabular}{|ll}
\hline F $\quad$ Singularities at $r=0$ & 38
\end{tabular}

\begin{tabular}{ll}
\hline G Prolate spherical coordinates & 39
\end{tabular}

\begin{tabular}{ll}
\hline H C-metric coordinates & 40
\end{tabular}

$\begin{array}{ll}\text { References } & 42\end{array}$ 


\section{Introduction}

Black holes in four-dimensional General Relativity have been the subject of intense research for several decades. One of the most important results on four-dimensional black holes in pure gravity, i.e. gravity without matter, is the uniqueness theorem stating that the rotating black hole solution of Kerr [1] is the unique solution for given mass and angular momentum [2, 3, 4, 5]. This shows that the phase structure of black holes in four dimensions is very simple: Only one phase is available.

In recent years, attention have turned to the study of black holes in higher-dimensional General Relativity. It is by now clear that the phase structure of black holes is much more complicated when having more than four dimensions. For five-dimensional asymptotically flat black hole solutions, it was discovered by Emparan and Reall in [6] that in addition to the Myers-Perry rotating black hole solution [7, which has horizon topology $S^{3}$, there exists also a rotating black ring solution with horizon topology $S^{2} \times S^{1}$. This means that for a given mass and angular momentum one can have as many as three different available phases, for five-dimensional asymptotically flat solutions of pure gravity. For pure gravity solutions asymptoting to Minkowski-space times a circle $\mathcal{M}^{d} \times S^{1}$, one has an even richer phase structure, involving phases with different horizon topologies and also phases with Kaluza-Klein bubbles. ${ }^{1}$

The complicated and rich phase structure of black holes in higher dimensions makes it desirable to develop new tools to find exact solutions. We focus in this paper on a particular class of solutions: Stationary and axisymmetric solutions of the vacuum Einstein equations in higher-dimensional General Relativity, i.e. in pure gravity. These solutions have $D-2$ commuting Killing vector fields where $D$ is the dimension of the space-time. In four dimensions, this class of solutions includes the Kerr black hole [1, while in five dimensions both the rotating black hole with horizon topology $S^{3}$ [7] and the rotating black ring with horizon topology $S^{2} \times S^{1}$ [6] are in this class.

We find in this paper a canonical form of the metric for stationary and axisymmetric solutions of the vacuum Einstein equations in higher-dimensional General Relativity. With the metric in the canonical form, the Einstein equations takes a remarkably simple form: They reduce effectively to a differential equation that can be seen as an axisymmetric $D-2$ by $D-2$ matrix field $G$ living in a three-dimensional flat space, apart from a subclass of solutions that instead reduce to a set of equations on a $D-2$ by $D-2$ matrix field living in two-dimensional flat space.

We analyze the general structure of such solutions. In the three-dimensional space that $G$ can be seen to live in the sources for $G$ are in the form of thin rods along a line. We examine the general structure of the rods that constitute the sources of a given solution. We furthermore identify the asymptotic behavior of asymptotically flat solutions in four and five dimensions.

\footnotetext{
${ }^{1}$ See [8 9] and references therein.
} 
As examples of stationary and axisymmetric solutions, we consider the five-dimensional rotating black hole with horizon topology $S^{3}$ and the black ring with horizon topology $S^{2} \times S^{1}$. We write down the metric in the canonical coordinates and analyze their rodstructure, i.e. the structure of their sources.

In four dimensions, the canonical form of the metric that we find for stationary and axisymmetric solutions is equivalent to the so-called Papapetrou form for the metric 10 , 11. Papapetrou found that, under certain conditions, the metric of four-dimensional stationary and axisymmetric pure gravity solutions can be written in the form ${ }^{2}$

$$
d s^{2}=-e^{2 U}(d t+A d \phi)^{2}+e^{-2 U} r^{2} d \phi^{2}+e^{2 \nu}\left(d r^{2}+d z^{2}\right) .
$$

The functions $U(r, z), A(r, z)$ are solutions of

$$
\left(\partial_{r}^{2}+\frac{1}{r} \partial_{r}+\partial_{z}^{2}\right) U=-\frac{e^{4 U}}{2 r^{2}}\left[\left(\partial_{r} A\right)^{2}+\left(\partial_{z} A\right)^{2}\right], \quad \partial_{r}\left(\frac{e^{4 U}}{r} \partial_{r} A\right)+\partial_{z}\left(\frac{e^{4 U}}{r} \partial_{z} A\right)=0,
$$

and the function $\nu(r, z)$ is a solution of

$$
\begin{gathered}
\partial_{r} \nu=-\partial_{r} U+r\left[\left(\partial_{r} U\right)^{2}-\left(\partial_{z} U\right)^{2}\right]-\frac{e^{4 U}}{4 r}\left[\left(\partial_{r} A\right)^{2}-\left(\partial_{z} A\right)^{2}\right] \\
\partial_{z} \nu=-\partial_{z} U+2 r \partial_{r} U \partial_{z} U-\frac{e^{4 U}}{2 r} \partial_{r} A \partial_{z} A .
\end{gathered}
$$

Here $\partial / \partial t$ and $\partial / \partial \phi$ are the two Killing vector fields. Since the equations (1.3) for $\nu$ are integrable, one can solve the Einstein equations by first finding $U$ and $A$ that solves (1.2), and then a $\nu$ can be found that solves (1.3).

The canonical form of the metric for stationary and axisymmetric solutions that we find in this paper is a generalization of the Papapetrou form (1.1) of the metric for fourdimensional solutions. Moreover, the simplified form of the Einstein equations that we find generalizes the equations (1.2)-(1.3) for four dimensions.

For the special case when all the $D-2$ Killing vector fields are orthogonal to each other, the canonical form of the metric that we find in this paper is equivalent to the form of the so-called generalized Weyl solutions of Emparan and Reall [16. ${ }^{3}$ In Ref. [16] it is shown that, under certain conditions, the metric for $D$-dimensional pure gravity solutions with $D-2$ commuting orthogonal Killing vector fields can be written in the form

$$
d s^{2}=-e^{2 U_{1}} d t^{2}+\sum_{i=2}^{D-2} e^{2 U_{i}}\left(d x^{i}\right)^{2}+e^{2 \nu}\left(d r^{2}+d z^{2}\right), \quad \sum_{i=1}^{D-2} U_{i}=\log r
$$

with $t=x^{1}$. The functions $U_{i}(r, z)$ are solutions of the three-dimensional Laplace equations

$$
\left(\partial_{r}^{2}+\frac{1}{r} \partial_{r}+\partial_{z}^{2}\right) U_{i}=0
$$

\footnotetext{
${ }^{2}$ See also [12 13, 14] 15].

${ }^{3}$ See [8] for a brief review of generalized Weyl solutions. See furthermore [17] for work on extending the generalized Weyl solutions of [16] to space-times with a cosmological constant.
} 
for $i=1, \ldots, D-2$, while $\nu(r, z)$ is a solution of

$$
\partial_{r} \nu=-\frac{1}{2 r}+\frac{r}{2} \sum_{i=1}^{D-2}\left[\left(\partial_{r} U_{i}\right)-\left(\partial_{z} U_{i}\right)^{2}\right], \quad \partial_{z} \nu=r \sum_{i=1}^{D-2} \partial_{r} U_{i} \partial_{z} U_{i}
$$

Here $\partial / \partial x^{i}, i=1, \ldots, D-2$, are the $D-2$ orthogonal Killing vector fields. Solutions with metric (1.4) with $U_{i}$ and $\nu$ obeying (1.5)-(1.6) are called generalized Weyl solutions.

We see that using the form of the metric (1.4) for solutions with $D-2$ commuting orthogonal Killing vector fields, solving the Einstein equations effectively reduces to the task of solving $D-3$ free Laplace equations on a three-dimensional flat space. This is due to the fact that the equations (1.6) for $\nu$ are integrable, so that one can find a $\nu$ solving (1.6) given any solution for $U_{i}, i=1, \ldots, D-2$.

It is important to remark that the method of generalized Weyl solutions generalizes Weyl's work on four-dimensional static and axisymmetric solutions [18. Moreover, one also obtains Weyl's form of the metric for four-dimensional static and axisymmetric solutions by setting $A=0$ in Papapetrou form (1.1). This is consistent with the fact that Eqs. (1.11)-(1.3) becomes equivalent to Eqs. (1.4)-(1.6) for $D=4$ when $A=0$, with $U_{1}=U$ and $U_{2}=\log r-U$.

Eqs. (1.5) can be seen as free Laplace equations for axisymmetric potentials living in a three-dimensional flat space. Solutions are then build up from thin rods located at the line $r=0$ in the three-dimensional space, with a given rod being a source for one of the $D-2$ potentials $U_{i}$ [16]. In this paper we generalize the concept of rods to the more general class of stationary and axisymmetric solutions, i.e. solutions for which the Killing vector fields are not necessarily orthogonal. One of the new features is that for a given rod we can associate a direction in the $(D-2)$-dimensional vector space spanned by the Killing vector fields. Solutions for which the directions of the rods are not orthogonal to each other are then also solutions where the Killing vector fields are not orthogonal to each other.

The outline of this paper is as follows: In Section 2 we derive a canonical form of the metric for stationary and axisymmetric pure gravity solutions. Using this, we find a simplified version of the Einstein equations, effectively reducing them to an equation on an axisymmetric $D-2$ by $D-2$ matrix field $G$ living in flat three-dimensional space. Some of the details of the derivation are placed in the Appendices $\mathrm{B}, \mathrm{C}$ and $\mathrm{D}$ In Appendix A we consider a special subclass of solutions that has the matrix field $G$ living in twodimensional flat space. In Appendix $\mathbb{E}$ we explore further the equation for the matrix field G.

In Section 3 we consider the behavior of the matrix field $G$ near the $r=0$ line in the flat three-dimensional space that $G$ lives in. The sources for $G$ lives on the $r=0$ line in the form of rods. We analyze the general structure of these rods. See also Appendix [F]

In Section 4 consider the asymptotic region, and we find out how to read off the asymptotic quantities for solutions that asymptotic to four-dimensional or five-dimensional 
Minkowski-space.

In Section [5] and 6] we write down the metrics for the five-dimensional rotating black hole of Myers and Perry and the rotating black ring of Emperan and Reall in the canonical form. We furthermore analyze the rod-structure for these solutions. For the rotating black hole solutions, we make use of Appendix G on prolate spherical coordinates, while for the black ring solutions we make use of Appendix $\mathrm{H}$ which considers $\mathrm{C}$-metric coordinates and how to transform these to the canonical coordinates of this paper.

In Section $\square$ we have the conclusions.

\section{Stationary and axisymmetric solutions}

In this section we show that finding stationary and axisymmetric solutions of General Relativity in $D$ dimensions without matter (i.e. pure gravity) can be reduced to finding solutions of a differential equation on an axisymmetric $D-2$ by $D-2$ matrix field in flat three-dimensional Euclidean space. As part of this, we find a particularly simple form of the metric for such solutions.

With respect to four-dimensional General Relativity, the results of this section generalizes the work of Papapetrou on stationary and axisymmetric metrics in four dimensions [10, 11] (see Eqs. (1.1)-(1.3) in the Introduction), which again is a generalization of the work of Weyl on static and axisymmetric metrics [18. In higher-dimensional General Relativity, the results of this section generalizes the work of Emparan and Reall on metrics with $D-2$ orthogonal commuting Killing vector fields [16] (see Eqs. (1.4)-(1.6) in the Introduction). We comment in more detail on the connection to previous work in the following. Finally, we note that the derivation of this section follows similar lines as that of Wald's derivation in [13] for four-dimensional stationary and axisymmetric metrics.

\subsection{Deriving canonical form of metric and the Einstein equations}

\section{Formulation of problem}

In this section we study $D$-dimensional manifolds which have $D-2$ commuting linearly independent Killing vector fields $V_{(i)}, i=1, \ldots, D-2$. With Lorentzian signature this corresponds to what we in this paper call stationary and axisymmetric space-times, where the term "stationary" means that one of our Killing vector fields are time-like, while the $D-3$ space-like Killing vector fields gives what we call "axisymmetry" of the space-time. ${ }^{4}$ That the Killing vector fields $V_{(i)}, i=1, \ldots, D-2$, commute means that

$$
\left[V_{(i)}, V_{(j)}\right]=0
$$

for $i, j=1, \ldots, D-2$. We see that the Killing vector fields generate a $(D-2)$-dimensional abelian group.

\footnotetext{
${ }^{4}$ One can use our results for null Killing vector fields, but we will not elaborate on that case in this paper.
} 
We restrict moreover ourselves to consider solutions of $D$-dimensional General Relativity without matter, i.e. we consider metrics that solve the vacuum Einstein equations

$$
R_{\mu \nu}=0 \text {. }
$$

In the following we find a canonical form of this class of metrics and we find furthermore a reduced form of the Einstein equations (2.2).

\section{Finding two-dimensional orthogonal subspaces}

Consider first a general $D$-dimensional space-time with $D-2$ commuting Killing vector fields $V_{(i)}, i=1, \ldots, D-2$. From the fact that the Killing vector fields are commuting, as expressed in Eq. (2.1), we get that we can find coordinates $x^{i}, i=1, \ldots, D-2$, and $u^{a}$, $a=1,2$, so that

$$
V_{(i)}=\frac{\partial}{\partial x^{i}}
$$

for $i=1, \ldots, D-2$. Clearly, this means that the metric components in this coordinate system only depends on $u^{1}$ and $u^{2}$.

We need now the theorem [13, 16]:

Theorem 2.1 Let $V_{(i)}, i=1, \ldots, D-2$, be $D-2$ commuting Killing vector fields such that:

(1) The tensor $V_{(1)}^{\left[\mu_{1}\right.} V_{(2)}^{\mu_{1}} \cdots V_{(D-2)}^{\mu_{D-2}} D^{\nu} V_{(i)}^{\rho]}$ vanishes at at least one point of the space-time for a given $i=1, \ldots, D-2$.

(2) The tensor $V_{(i)}^{\nu} R_{\nu}^{[\rho} V_{(1)}^{\mu_{1}} V_{(2)}^{\mu_{1}} \cdots V_{(D-2)}^{\left.\mu_{D-2}\right]}=0$ for all $i=1, \ldots, D-2$.

Then the two-planes orthogonal to the Killing vector fields $V_{(i)}, i=1, \ldots, D-2$, are integrable.

This theorem is stated and proven in four dimensions in [13] using Frobenius theorem on integrable submanifolds. Emparan and Reall generalized it to higher-dimensional manifolds in [16].

Assume now that the two conditions in Theorem 2.1 are obeyed. That the two-planes orthogonal to the Killing vector fields $V_{(i)}, i=1, \ldots, D-2$, are integrable means that for any given point of our $D$-dimensional manifold we have a two-dimensional submanifold that includes this point and moreover have the property that for any point of the submanifold the two-dimensional tangent-space is orthogonal to all of the Killing vector fields. By choosing coordinates on one of these two-dimensional submanifolds and dragging them along the integral curves of our Killing vector fields, we can find two coordinates $y^{1}$ and $y^{2}$ for our $D$-dimensional manifold so that $\frac{\partial}{\partial x^{i}}$ is orthogonal to $\frac{\partial}{\partial y^{a}}$ everywhere for all $i=1, \ldots, D-2$ and $a=1,2$. This means the metric takes the form

$$
d s^{2}=\sum_{i, j=1}^{D-2} G_{i j} d x^{i} d x^{j}+\sum_{a, b=1}^{2} \hat{g}_{a b} d y^{a} d y^{b}
$$


where $G_{i j}$ and $\hat{g}_{a b}$ only depends on $y^{1}$ and $y^{2}$.

From now on we restrict ourselves to solutions solving the vacuum Einstein equations (2.2). This ensures immediately that Condition (2) in Theorem 2.1 is obeyed. We assume furthermore that Condition (1) in Theorem 2.1] is obeyed. Condition (1) can for example be argued to hold if one of the Killing vector fields is an angle, since then it is zero on the axis of rotation. This means for instance that solutions asymptoting to Minkowski-space $\mathcal{M}^{D}$ for $D=4,5$ obeys Condition (1) since they have angles in them. Clearly, the same is true for solutions asymptoting to $\mathcal{M}^{D-p} \times T^{p}$ for $D-p=4,5$.

\section{The $r$ and $z$ coordinates}

Define now the function $r\left(y^{1}, y^{2}\right)$ as

$$
r=\sqrt{\left|\operatorname{det}\left(G_{i j}\right)\right|} .
$$

In Appendix $\mathrm{A}$ we treat the case in which $\operatorname{det}\left(G_{i j}\right)$ is constant, giving rise to a special class of solutions. Instead, we assume here and in the following that $r\left(y^{1}, y^{2}\right)$ is not a constant function. From Appendix $\mathbb{B}$ we get then that $\left(\frac{\partial r}{\partial y^{1}}, \frac{\partial r}{\partial y^{2}}\right) \neq(0,0)$ except in isolated points. We can then use the result of Appendix [C] that we can find a coordinate $z\left(y^{1}, y^{2}\right)$, along with two functions $\nu\left(y^{1}, y^{2}\right)$ and $\Lambda\left(y^{1}, y^{2}\right)$, so that

$$
\sum_{a, b=1}^{2} \hat{g}_{a b} d y^{a} d y^{b}=e^{2 \nu}\left(d r^{2}+\Lambda d z^{2}\right) .
$$

Therefore, the full metric takes the form

$$
d s^{2}=\sum_{i, j=1}^{D-2} G_{i j} d x^{i} d x^{j}+e^{2 \nu}\left(d r^{2}+\Lambda d z^{2}\right),
$$

where $\nu(r, z)$ and $\Lambda(r, z)$ are functions of $r$ and $z$.

From Appendix D, where part of the Ricci tensor for the metric (2.7) is computed, we have from Eq. (D.8)

$$
\sum_{i, j=1}^{D-2} G^{i j} R_{i j}=-\frac{\partial_{r} \Lambda}{2 e^{2 \nu} \Lambda r} .
$$

Since our solution should fulfil the vacuum Einstein equations $R_{\mu \nu}=0$, this means that

$$
\partial_{r} \Lambda=0
$$

This gives that $\Lambda=\Lambda(z)$. Since we preserve the form of the metric (2.7) under a transformation $z^{\prime}=f(z)$ we can therefore set $\Lambda(z)=1$ by a coordinate transformation of $z$ alone. Thus, we can define the $z$-coordinate by demanding $\Lambda=1$. This fixes $z$ up to transformations $z \rightarrow z+$ constant. 


\section{Canonical form of metric}

In conclusion, we have shown that for any Ricci-flat space-time with $D-2$ commuting Killing vector fields $V_{(i)}, i=1, \ldots, D-2$, obeying Condition (1) of Theorem 2.1, we can find a coordinate system $\left(x^{1}, \ldots, x^{D-2}, r, z\right)$ such that $V_{(i)}=\frac{\partial}{\partial x^{i}}$ and such that the metric takes the canonical form

$$
d s^{2}=\sum_{i, j=1}^{D-2} G_{i j} d x^{i} d x^{j}+e^{2 \nu}\left(d r^{2}+d z^{2}\right)
$$

with

$$
r=\sqrt{\left|\operatorname{det}\left(G_{i j}\right)\right|},
$$

where $G_{i j}(r, z)$ and $\nu(r, z)$ are functions only of $r$ and $z$. In addition to the assumption that the Killing vector fields should obey Condition (1) of Theorem 2.1 we also assume here that $\operatorname{det}\left(G_{i j}\right)$ is not constant on our space-time. The situation in which $\operatorname{det}\left(G_{i j}\right)$ is constant is instead treated in Appendix A.

\section{The Einstein equations}

We now consider the vacuum Einstein equations $R_{\mu \nu}=0$ for the metric (2.10) with the constraint (2.11) using the computed Ricci tensor (D.9) in Appendix D.

Considering the $R_{i j}=0$ equations we see from (D.9) that the equations for $G_{i j}$ are

$$
\left(\partial_{r}^{2}+\frac{1}{r} \partial_{r}+\partial_{z}^{2}\right) G_{i j}=\sum_{k, l=1}^{D-2} G^{k l} \partial_{r} G_{k i} \partial_{r} G_{l j}+\sum_{k, l=1}^{D-2} G^{k l} \partial_{z} G_{k i} \partial_{z} G_{l j}
$$

Considering the $R_{r r}-R_{z z}=0$ and $R_{r z}=0$ equations we see from (D.9) that the equations for $\nu$ are

$$
\begin{gathered}
\partial_{r} \nu=-\frac{1}{2 r}+\frac{r}{8} \sum_{i, j, k, l=1}^{D-2} G^{i j} G^{k l} \partial_{r} G_{i k} \partial_{r} G_{j l}-\frac{r}{8} \sum_{i, j, k, l=1}^{D-2} G^{i j} G^{k l} \partial_{z} G_{i k} \partial_{z} G_{j l}, \\
\partial_{z} \nu=\frac{r}{4} \sum_{i, j, k, l=1}^{D-2} G^{i j} G^{k l} \partial_{r} G_{i k} \partial_{z} G_{j l} .
\end{gathered}
$$

Using now (2.13) together with (2.12) one can check that the integrability condition $\partial_{z} \partial_{r} \nu=\partial_{r} \partial_{z} \nu$ on $\nu(r, z)$ is obeyed. Thus, for a given solution $G_{i j}(r, z)$ of (2.12) the equations (2.13) can be integrated to give $\nu(r, z)$.

Finally, there is the remaining non-trivial equation $R_{r r}+R_{z z}=0$ coming from the Einstein equations. The explicit expression for this equation is easily found using (D.9) and is seen to involve second derivatives of $\nu$. Since $G_{i j}(r, z)$ and $\nu(r, z)$ already are determined by (2.12)-(2.13) it needs to be checked that $R_{r r}+R_{z z}=0$ is consistent with (2.12)-(2.13). This can be checked by finding $\partial_{r}^{2} \nu+\partial_{z}^{2} \nu$ from (2.13). Inserting the result into $R_{r r}+R_{z z}$ from (D.9) this is seen to be zero using (2.12). 
Therefore, we have shown that one can find solutions of the vacuum Einstein equations for the canonical form for the metric (2.10)-(2.11) by finding a $G_{i j}(r, z)$ that satisfies (2.12). Then, subsequently one can always find a function $\nu(r, z)$ that satisfies (2.13), and thereby we have a complete solution satisfying all the Einstein equations.

\section{Reduction to Papapetrou form and generalized Weyl solutions}

We show here that the canonical form of the metric (2.10)-2.11), along with the form of the Einstein equations (2.12)-(2.13), reduces to the previously known cases.

We first consider the Papapetrou form (1.1) for four dimensional stationary and axisymmetric solutions [10, 11], with the Einstein equations in the form (1.2)-(1.3). Setting $D=4$, we see that by setting $G_{11}=-e^{2 U}, G_{12}=-e^{2 U} A$ and $G_{22}=e^{-2 U}\left(r^{2}-A^{2} e^{4 U}\right)$ with $x^{1}=t$ and $x^{2}=\phi$, we get the Papapetrou form (1.1) from (2.10). Furthermore, we see that (2.12)-(2.13) reduce to (1.2)-(1.3).

Consider now instead the generalized Weyl solutions of [16] which have $D-2$ orthogonal commuting Killing vector fields. These have metric (1.4), and the Einstein equations are in the form (1.5)-(1.6). We see that setting $G_{11}=-e^{2 U_{1}}$ and $G_{i i}=e^{2 U_{i}}$ for $i=2, . ., D-2$, we get the metric (1.4) from the canonical form (2.10). $\operatorname{det} G=-r^{2}$ gives then $\sum_{i=1}^{D-2} U_{i}=$ $\log r$. For the Einstein equations, it is easily seen that (2.12)-(2.13) reduces to (1.5)-(1.6) (see also Appendix E). Thus, the canonical form (2.10)-(2.11) correctly reduce to the generalized Weyl solutions.

\subsection{Compact notation for the equations for $G_{i j}(r, z)$}

We have derived above that the metric of $D$-dimensional manifolds with $D-2$ commuting Killing vector fields obeying the vacuum Einstein equations can be written in the canonical form (2.10)-(2.11). Moreover, the vacuum Einstein equations reduce to (2.12)-(2.13). We now show that we can write the equations for $G_{i j}(r, z)$ in a more compact form. This is highly useful for analysis of these equations.

For a given $r$ and $z$ we can view $G_{i j}$ as a $D-2$ times $D-2$ real symmetric matrix, with $G^{i j}$ as its inverse. In this way we can write (2.12) in matrix notation as

$$
G^{-1}\left(\partial_{r}^{2}+\frac{1}{r} \partial_{r}+\partial_{z}^{2}\right) G=\left(G^{-1} \partial_{r} G\right)^{2}+\left(G^{-1} \partial_{z} G\right)^{2},
$$

with the constraint $|\operatorname{det} G|=r^{2}$ coming from (2.11).

We can make a further formal rewriting of (2.12) by recognizing that the derivatives respects the symmetries of a flat three-dimensional Euclidean space with metric

$$
d r^{2}+r^{2} d \gamma^{2}+d z^{2}
$$

Here $\gamma$ is an angular coordinate of period $2 \pi .{ }^{5}$ Therefore, if we define $\vec{\nabla}$ to be the gradiant

\footnotetext{
${ }^{5}$ It is important to remark that $\gamma$ is not an actual physical variable for the solution [2.10), but rather an auxilirary coordinate that is useful for understanding the structure of Eqs. (2.12).
} 
in three-dimensional flat Euclidean space, we can write (2.12) as

$$
G^{-1} \vec{\nabla}^{2} G=\left(G^{-1} \vec{\nabla} G\right)^{2}
$$

Thus, by finding the axisymmetric solutions of the differential matrix equation (2.16) in three-dimensional flat Euclidean space, that obey the constraint $|\operatorname{det} G|=r^{2}$, we can find all stationary and axisymmetric solutions of the vacuum Einstein equations in $D$ dimensions. ${ }^{6}$

We explore some of the mathematical properties of Eq. (2.16) in Appendix E Here the compact form (2.16) of (2.12) prove highly useful.

\section{Behavior of solutions near $r=0$}

In Section 2 we derived the canonical form of the metric (2.10)-(2.11), along with the corresponding EOMs (2.12)-(2.13), for stationary and axisymmetric solutions of the vacuum Einstein equations. In this section we consider the behavior of such solutions close to $r=0$.

\subsection{Behavior of $G(r, z)$ near $r=0$}

We describe first how the $z$-axis at $r=0$ is divided into intervals, called rods, according to the dimension of the kernel of $G$ at $r=0$. We find then coordinates in which a solution simplifies near a rod, making it possible to describe the solution in detail near the rod. We use this to define the rod-structure of a solution in Section 3.2

\section{Dividing the $z$-axis into rods}

Consider a given solution $G(r, z) . G(r, z)$ is required to be continuous. Since $|\operatorname{det} G|=r^{2}$ we see that the product of the eigenvalues of $G(r, z)$ goes to zero for $r \rightarrow 0$. Therefore, we have that the eigenvalues of $G(0, z)$, which all are real since $G(0, z)$ is symmetric, include the eigenvalue zero for a given $z$. This means that the dimension of the kernel of $G(0, z)$ is greater than or equal to one for any $z$. We can write this more compactly as $\operatorname{dim}(\operatorname{ker}(G(0, z))) \geq 1$.

A necessary condition for a regular solution is that precisely one eigenvalue of $G(0, z)$ is zero for a given $z$, except in isolated points. This statement is explained in Appendix [F] where we argue that if we have more than one eigenvalue going to zero as $r \rightarrow 0$, for a given $z$, we have a curvature singularity at that point. Therefore, in the following we consider only solutions which, for a given $z$, only have one eigenvalue going to zero for $r \rightarrow 0$, except at isolated values of $z$. Written compactly, this means $\operatorname{dim}(\operatorname{ker}(G(0, z))=1$,

\footnotetext{
${ }^{6}$ To be precise, we mean all solutions for which the $D-2$ Killing vector fields obey Condition (1) of Theorem 2.1 and for which $\operatorname{det}\left(G_{i j}\right)$ is a non-constant function on our $D$-dimensional manifold.
} 
except at isolated values of $z$. Denote now these isolated values of $z$ as $a_{1}, a_{2}, \ldots, a_{N}$, with $a_{1}<a_{2}<\cdots<a_{N}$.

We see now that we divided the $z$-axis into the $N+1$ intervals $\left[-\infty, a_{1}\right],\left[a_{1}, a_{2}\right], \ldots$, $\left[a_{N-1}, a_{N}\right]$ and $\left[a_{N}, \infty\right] .{ }^{7}$ We call these $N+1$ intervals the rods of the solution.

One can easily check that the above definition of rods reduces to the definition of [16] for the special case of generalized Weyl solutions, i.e. with $D-2$ orthogonal Killing vector fields.

\section{Behavior of $G(r, z)$ near a rod}

In Section 2 we found that $G(r, z)$ should solve the equation $G^{-1} \vec{\nabla}^{2} G=\left(G^{-1} \vec{\nabla} G\right)^{2}$ with the constraint that $|\operatorname{det} G|=r^{2}$. However, this breaks down as $r \rightarrow 0$, because for $r=0$ we have that $\operatorname{det} G=0$ so $G$ is not invertible anymore. The reason for this is that we have sources added to the equation $G^{-1} \vec{\nabla}^{2} G=\left(G^{-1} \vec{\nabla} G\right)^{2}$ at $r=0$. The sources corresponds precisely to the rods defined above, i.e. the intervals with $\operatorname{dim}(\operatorname{ker}(G(r, z)))=1$. Moreover, if we view the solution $G(r, z)$ as a matrix-valued field in the unphysical three-dimensional flat Euclidean space with metric (2.15), a rod is really a source in the form of a rod of zero thickness in this unphysical space. In the following we examine in detail the equation $G^{-1} \vec{\nabla}^{2} G=\left(G^{-1} \vec{\nabla} G\right)^{2}$ near a rod in order to describe more precisely the behavior of $G(r, z)$ near a rod.

Consider a solution $G(r, z)$ and a given $\operatorname{rod}\left[z_{1}, z_{2}\right]$. Consider furthermore a given value of $z=z_{*}$ obeying $z_{1}<z_{*}<z_{2}$. Since $G\left(0, z_{*}\right)$ is a symmetric real matrix we can diagonalize it using an orthogonal matrix $\Lambda_{*}$ such that $\Lambda_{*}^{T} G\left(0, z_{*}\right) \Lambda_{*}$ is diagonal. Furthermore, since $G\left(0, z_{*}\right)$ has precisely one zero eigenvalue, we can choose $\Lambda_{*}$ so that $\left(\Lambda_{*}^{T} G\left(0, z_{*}\right) \Lambda_{*}\right)_{11}=0$.

Define now $\tilde{G}(r, z)=\Lambda_{*}^{T} G(r, z) \Lambda_{*}$. Clearly, $\tilde{G}(r, z)$ is a solution of (2.12) by Lemma E.7 and furthermore $\operatorname{det} \tilde{G}=\operatorname{det} G= \pm r^{2}$. Note that all entries of $\tilde{G}\left(r, z_{*}\right)$ are of order $\mathcal{O}\left(r^{2}\right)$ for $r \rightarrow 0$, expect the entries $\tilde{G}_{i i}\left(r, z_{*}\right), i=2, \ldots, D-2$, which are finite and non-zero.

Since $\tilde{G}^{i j}\left(0, z_{*}\right)$ is not well-defined we need to consider the limit $\tilde{G}^{i j}\left(r, z_{*}\right)$ for $r \rightarrow 0$ carefully. To this end, define the $D-2$ by $D-2$ matrix-valued function $M(r, z)$ by

$$
M_{11}=\frac{\tilde{G}_{11}}{r^{2}}, \quad M_{1 i}=\frac{\tilde{G}_{1 i}}{r}, \quad M_{i j}=\tilde{G}_{i j}, \quad i, j=2, \ldots, D-2
$$

for any $(r, z)$. We see that this corresponds to a rescaling $x_{\text {new }}^{1}=r x_{\text {old }}^{1}$. Clearly, we have that $M\left(0, z_{*}\right)$ is diagonal, with non-zero eigenvalues. Moreover, we have that $\tilde{G}^{11}=$ $M^{11} / r^{2}, \tilde{G}^{1 i}=M^{1 i} / r$ and $\tilde{G}^{i j}=M^{i j}, i, j=2, \ldots, D-2$. Since $M_{1 i}, i=2, . ., D-2$, are of order $\mathcal{O}(r)$, we have that $M^{1 i}, i=2, . ., D-2$, are of order $\mathcal{O}(r)$, and therefore that $\tilde{G}^{1 i}\left(r, z_{*}\right), i=2, . ., D-2$, stay finite (or goes to zero) in the limit $r \rightarrow 0$. Also, $\tilde{G}^{i j}\left(r, z_{*}\right) \rightarrow 0$ with $2 \leq i<j \leq D-2$ and $\tilde{G}^{i i}\left(r, z_{*}\right) \rightarrow\left(\tilde{G}_{i i}\left(0, z_{*}\right)\right)^{-1}$ with $i=2, \ldots, D-2$, while $\tilde{G}^{11}\left(r, z_{*}\right)$ is of order $1 / r^{2}$ for $r \rightarrow 0$.

\footnotetext{
${ }^{7}$ Note that it is possible to have an infinite number of intervals.
} 
Consider now the equation $\tilde{G}^{-1} \vec{\nabla}^{2} \tilde{G}=\left(\tilde{G}^{-1} \vec{\nabla} \tilde{G}\right)^{2}$ for $z=z_{*}$ and $r \rightarrow 0$. We have

$$
\vec{\nabla}^{2} \tilde{G}_{11}=\tilde{G}^{11}\left(\vec{\nabla} \tilde{G}_{11}\right)^{2}+2 \sum_{i=2}^{D-2} \tilde{G}^{1 i} \vec{\nabla} \tilde{G}_{11} \cdot \vec{\nabla} \tilde{G}_{1 i}+\sum_{i=2}^{D-2} \tilde{G}^{i i}\left(\vec{\nabla} \tilde{G}_{1 i}\right)^{2}
$$

up to terms that go to zero for $r \rightarrow 0$. Since we just found that $\tilde{G}^{1 i}$ and $\tilde{G}^{i i}$ are finite for $z=z_{*}$ and $r \rightarrow 0$ (with $i=2, \ldots, D-2$ ) and since we require that $\tilde{G}_{i j}(r, z)$ and its derivatives are finite as a necessary condition for regular solutions, ${ }^{8}$ we see that the LHS and the second and third term on the RHS of (3.2) are finite for $z=z_{*}$ and $r \rightarrow 0$. Since $\tilde{G}^{11} \rightarrow \infty$ we see therefore that we need $\vec{\nabla} \tilde{G}_{11} \rightarrow 0$ for $z=z_{*}$ and $r \rightarrow 0$. If we consider instead $\vec{\nabla}^{2} \tilde{G}_{i i}$ we see similarly that

$$
\vec{\nabla}^{2} \tilde{G}_{i i}=\tilde{G}^{11}\left(\vec{\nabla} \tilde{G}_{1 i}\right)^{2}+\text { finite terms }
$$

so we get that $\vec{\nabla} \tilde{G}_{1 i} \rightarrow 0$ for $z=z_{*}$ and $r \rightarrow 0$. Thus, we have derived that $\vec{\nabla} \tilde{G}_{1 i}\left(0, z_{*}\right)=0$ for $i=1, \ldots, D-2$. In particular, this implies that $\partial_{z} \tilde{G}_{1 i}\left(0, z_{*}\right)=0$. Therefore, since this works for any $\left.z_{*} \in\right] z_{1}, z_{2}$ [ we get the following theorem:

Theorem 3.1 Consider a rod $\left[z_{1}, z_{2}\right]$ for a solution $G(r, z)$. Then we can find an orthogonal matrix $\Lambda_{*}$ such that the solution $\tilde{G}(r, z)=\Lambda_{*}^{T} G(r, z) \Lambda_{*}$ has the property that $\tilde{G}_{1 i}(0, z)=0$ for $i=1, \ldots, D-2$ and $z \in\left[z_{1}, z_{2}\right]$.

We take now a closer look at the EOMs (2.12)-(2.13) near $r=0$. Consider a solution $G(r, z)$ and a particular $\operatorname{rod}\left[z_{1}, z_{2}\right]$. Using Theorem 3.1 we always make a constant coordinate transformation of the $x^{i}$ coordinates so that $G(r, z)$ has the property that $G_{1 i}(0, z)=0$ for $i=1, \ldots, D-2$ and $z \in\left[z_{1}, z_{2}\right]$. To leading order, we can therefore write $G(r, z)=\left( \pm a(z) r^{2}\right) \oplus A(z)$ for $r \rightarrow 0$ with $z_{1}<z<z_{2}$ where $a(z)$ is a function of $z$ with $a(z)>0$ for $z \in] z_{1}, z_{2}[$ and $A(z)$ is a $D-3$ by $D-3$ matrix-valued function of $z$. Thus, $G_{11}= \pm a(z) r^{2}$ for $r \rightarrow 0$. Note that $|\operatorname{det}(A(z))|=1 / a(z)$.

If we consider Eqs. (2.12) we see that $\vec{\nabla}^{2} G_{11}= \pm 4 a(z)+\mathcal{O}(r)$ and that $G^{11}\left(\partial_{r} G_{11}\right)^{2}=$ $\pm 4 a(z)+\mathcal{O}(r)$, so this is consistent. Considering $\partial_{r} \nu$ in (2.13) we see that since $\partial_{r} G_{11}=$ $\pm 2 a(z) r$ we have that $\partial_{r} \nu=0$ to leading order. Considering instead $\partial_{z} \nu$ in (2.13), we get

$$
\partial_{z} \nu=\frac{1}{2} \frac{a^{\prime}}{a}+\mathcal{O}(r)
$$

Thus, to leading order for $r \rightarrow 0$ we have $e^{2 \nu}=c^{2} a(z)$ where $c$ is a positive number. Therefore, for $r \rightarrow 0$ with $z_{1}<z<z_{2}$ the metric (2.10) has the form

$$
d s^{2}=\sum_{i, j=2, \ldots, D-2} A_{i j}(z) d x^{i} d x^{j}+a(z)\left[ \pm r^{2}\left(d x^{1}\right)^{2}+c^{2}\left(d r^{2}+d z^{2}\right)\right] .
$$

This is the behavior of the canonical metric (2.10) near a rod.

\footnotetext{
${ }^{8}$ Except in the endpoints of a rod where the derivatives are not necessarily well-defined. Hence the condition $z_{1}<z_{*}<z_{2}$.
} 
Notice now that if $G_{11} / r^{2}$ is positive for $r \rightarrow 0$ the coordinate $x^{1}$ is space-like and the metric (3.5) has a conical singularity for $r \rightarrow 0$, unless $x^{1}$ is periodic with period $2 \pi c$. For a regular solution, this means that if we have a rod in a space-like direction we have necessarily that this direction is periodic with the period constrained from avoiding the conical singularity.

If $G_{11} / r^{2}$ is negative for $r \rightarrow 0$ the coordinate $x^{1}$ is time-like and we see that there is a horizon at $r=0$ since $G_{11}=0$. Moreover, using the above argument for the space-like direction, we see that the Wick rotated coordinate $i x^{1}$ must be periodic with period $2 \pi c$. This means that the horizon has a temperature $T=1 /(2 \pi c)$ associated to it.

\subsection{The rod-structure of a solution}

In this section we define what we mean by the rod-structure of a solution, and we discuss the general structure of rods, in view of the considerations of Section 3.1.

\section{Specifying the rod-structure of a solution}

Let a solution $G_{i j}(r, z)$ of Eqs. (2.11)-(2.12) be given with $N+1$ rods which meet in the $z$-values $a_{1}<a_{2}<\ldots<a_{N}$. Introduce here the notation $a_{0}=-\infty$ and $a_{N+1}=\infty$ in order to write the equations below more compactly. The solution $G(r, z)$ thus have the $N+1 \operatorname{rods}\left[a_{k-1}, a_{k}\right]$ with $k=1, \ldots, N+1$.

Define now for the solution $G_{i j}(r, z)$ the $N+1$ vectors $v_{(k)}$ in $\mathbb{R}^{D-2}, k=1, \ldots, N+1$, by

$$
G(0, z) v_{(k)}=0 \text { for } z \in\left[a_{k-1}, a_{k}\right] \quad, \quad k=1, \ldots, N+1,
$$

with $v_{(k)} \neq 0$ for all $k=1, \ldots, N+1$. In other words, $v_{(k)} \in \operatorname{ker}(G(0, z))$. We call $v_{(k)}$ the direction of the corresponding rod $\left[a_{k-1}, a_{k}\right]$.

We define then the rod-structure of the solution $G_{i j}(r, z)$ as the specification of the rod intervals $\left[a_{k-1}, a_{k}\right]$ plus the corresponding directions $v_{(k)}, k=1, \ldots, N+1$.

Obviously, since $v_{(k)}$ is defined as an eigenvector, it is only defined up to a multiplicative factor (different from zero). In other words, one should really regard $v_{(k)}$ as an element of the real projective space $\mathbb{R} P^{D-3}$.

We now demonstrate that it follows from the considerations of Section 3.1 that the above definition of the rod-structure is meaningfull. This involves showing that $v_{(k)}$ as defined in (3.6) exists and is unique, as element in $\mathbb{R} P^{D-3}$

Observe first that by Theorem 3.1 we get for each of the $N+1$ rods an orthogonal

matrix $\Lambda_{(k)}, k=1, \ldots, N+1$, so that $\left(\Lambda_{(k)}^{T} G(0, z) \Lambda_{(k)}\right)_{1 i}=0$ for $z \in\left[a_{k-1}, a_{k}\right]$. Define the unit vector $e=(1,0, \ldots, 0)$ in $\mathbb{R}^{D-2}$. Note that from the above we have then that $\Lambda_{(k)}^{T} G(0, z) \Lambda_{(k)} e=0$ for $z \in\left[a_{k-1}, a_{k}\right]$ with $k=1, \ldots, N+1$. We can now define the $N+1$ vectors $v_{(k)}=\Lambda_{(k)} e, k=1, \ldots, N+1$. Clearly, then these $N+1$ vectors $v_{(k)}$ obey (3.6). Thus, we have shown that we can always find $N+1$ vectors $v_{(k)}$ obeying (3.6). 
To see that each of the $N+1$ vectors $v_{(k)}$ are unique, seen as elements of $\mathbb{R} P^{D-3}$, it is enough to notice that we know from Section 3.1 that $\operatorname{dim}(\operatorname{ker}(G(0, z)))=1$ for $z \in] a_{k-1}, a_{k}[$.

\section{Discussion of existence and uniqueness of solutions}

We discuss here whether a solution is uniquely given by its rod-structure, and whether there exists a solution for any given rod-structure. We consider here only solutions of Euclidean signature, but one can easily extend the considerations to solutions of Lorentzian signature.

If we consider the special case of the generalized Weyl solutions of [16], corresponding to $G(r, z)$ being a diagonal matrix, we clearly have the directions of the rods can be chosen to have the form $v_{(k)}=(0, \ldots, 0, \pm 1,0, \ldots, 0)$. It is then known from the analysis of [16] that we can specify a solution completely by the $N$ parameters $a_{1}<\ldots<a_{N}$ and $N+1$ vectors $v_{(k)}$, i.e. a solution is completely specified by its rod-structure.

We now speculate that this statement can be generalized, i.e. that also in the more general class of solution considered here, a solution is specified uniquely by its rod-structure. Thus, we claim in detail that: A solution with $N+1$ rods is completely determined by specifying the parameters $a_{1}<\ldots<a_{N}$ and directions $v_{(k)}, k=1, \ldots, N+1$. I.e. it is not possible to find two physically different solutions with $N+1$ rods that have the same $N$ parameters $a_{k}$ and $N+1$ directions $v_{(k)}$. Intuitively, this statement seems valid since one would expect that the system of equations (2.12) determine $G(r, z)$ once we have determined the sources for $G(r, z)$ at $r=0$. And, the values $a_{k}$ and directions $v_{(k)}$ seems precisely to specify that.

Note that if this statement is true, it is moreover true that it is not possible to find two physically different solutions with $N+1$ rods that have the same $N$ parameters $a_{k}$, up to a global translation of all $N$ parameters, and the same $N+1$ directions $v_{(k)}$, up to a global rotation of all $N+1$ directions.

One can also turn things around and ask whether there exists a solution with $N+1$ rods given the $N$ parameters $a_{1}<\ldots<a_{N}$ and $N+1$ directions $v_{(k)}, k=1, \ldots, N+1$ (not imposing the solution to be regular). This would be interesting to examine further. However, there is an obvious restriction on the directions of the first and last rod $\left[-\infty, a_{1}\right]$ and $\left[a_{N}, \infty\right]$. For a given asymptotic space, which the solution is required to asymptote to for $\sqrt{r^{2}+z^{2}} \rightarrow \infty$ with $z / \sqrt{r^{2}+z^{2}}$ fixed (see Section 4), the directions of these two rods should be correlated, and can therefore not be chosen independently.

\subsection{Analysis of the rod-structure}

In this section we summarize how to analyze the rod-structure, and add some useful nomenclature and general comments. We consider here solutions $G_{i j}(r, z)$ of (2.12) with $\operatorname{det} G=r^{2}$. 
In Section 3.1 we learned that in order to avoid curvature singularities it is a necessary condition on a solution that the eigenspace for the eigenvalue zero of the matrix $G(0, z)$ for a given $z$ should be one-dimensional, except for isolated values of $z$. We therefore restrict ourselves to solutions where this applies. Naming the isolated $z$-values as $a_{1}, \ldots, a_{N}$, we see that the $z$-axis splits up into the $N+1$ intervals $\left[-\infty, a_{1}\right],\left[a_{1}, a_{2}\right], \ldots,\left[a_{N-1}, a_{N}\right],\left[a_{N}, \infty\right]$. The first task in understanding the rod-structure of a solution is thus to find these intervals, called rods.

Consider now a specific rod $\left[z_{1}, z_{2}\right]$. From Theorem 3.1 (see also Section 3.2) we know that we can find a vector

$$
v=v^{i} \frac{\partial}{\partial x^{i}}
$$

so that

$$
\sum_{j=1}^{D-2} G_{i j}(0, z) v^{j}=0,
$$

for $i=1, \ldots, D-2$ and $z \in\left[z_{1}, z_{2}\right]$. This vector $v$ is called the direction of the $\operatorname{rod}\left[z_{1}, z_{2}\right]$. Then, if $G_{i j} v^{i} v^{j} / r^{2}$ is negative (positive) for $r \rightarrow 0$ we say the rod $\left[z_{1}, z_{2}\right]$ is time-like (space-like).

Consider now a space-like rod $\left[z_{1}, z_{2}\right]$. For $r \rightarrow 0$ with $\left.z \in\right] z_{1}, z_{2}[$ we have a potential conical singularity. Let $\eta$ be a coordinate, made as a linear combination of $x^{i}, i=1, \ldots, D-$ 2 , with

$$
\frac{\partial}{\partial \eta}=v=v^{i} \frac{\partial}{\partial x^{i}}
$$

Then in order to cure the conical singularity at the rod, the coordinate $\eta$ should have period

$$
\Delta \eta=2 \pi \lim _{r \rightarrow 0} \sqrt{\frac{r^{2} e^{2 \nu}}{G_{i j} v^{i} v^{j}}},
$$

with $z \in\left[z_{1}, z_{2}\right]$. This is seen from the analysis of Section 3.1. We see from this that a space-like rod corresponds to a compact direction. For a time-like rod, one can similarly find an associated temperature, by doing a Wick rotation. Therefore, a time-like rod corresponds to a horizon (see Section 3.1).

We introduce here some additional nomenclature for rods. Consider a rod $\left[z_{1}, z_{2}\right]$. If this is a finite interval we call $\left[z_{1}, z_{2}\right]$ a finite rod. If either $z_{1}=-\infty$ or $z_{2}=\infty$ but not both of them, we call $\left[z_{1}, z_{2}\right]$ a semi-infinite rod. $[-\infty, \infty]$ is instead called the infinite rod.

As discussed in [16], a finite time-like rod corresponds to an event horizon, at least if there are no semi-infinite time-like rods for the solution. Similarly, a finite space-like rod corresponds to a Kaluza-Klein direction if there are no semi-infinite space-like rods in that direction. Moreover, a (semi-)infinite space-like rod corresponds to an axis of rotation, with the associated coordinate being the rotation angle, while a semi-infinite time-like rod corresponds to an acceleration horizon. 


\section{Asymptotically flat space-times}

In this section we consider asymptotically flat space-times. We consider the four- and five-dimensional Minkowski-spaces $\mathcal{M}^{4}$ and $\mathcal{M}^{5}$, and we consider the asymptotic behavior of solutions that asymptote to $\mathcal{M}^{4}$ and $\mathcal{M}^{5}$.

The Minkowski-spaces $\mathcal{M}^{4}$ and $\mathcal{M}^{5}$ are special in that they are the only Minkowskispaces that one can describe using the ansatz (2.10) -(2.11). This is easily seen by counting the number of Killing vector fields. An obvious generalization of the considerations of this section would be to consider the Kaluza-Klein space-times $\mathcal{M}^{4} \times S^{1}$ and $\mathcal{M}^{5} \times S^{1}$, or other space-times with even more compact directions, i.e. $\mathcal{M}^{4} \times T^{p}$ or $\mathcal{M}^{5} \times T^{p}$. We leave this for the future. ${ }^{9}$

In the following we put Newtons constant $G_{\mathrm{N}}=1$. To reinstate $G_{\mathrm{N}}$ one should substitute $M \rightarrow G_{\mathrm{N}} M$ and $J \rightarrow G_{\mathrm{N}} J$.

\subsection{Perturbation of diagonal metric}

Before describing asymptotically flat spaces, we first develop a tool that will prove useful. We consider in this section a perturbation $\delta G(r, z)$ of a solution $G(r, z)$ of Eqs. (2.12), with $G(r, z)$ being diagonal, such that $G(r, z)+\delta G(r, z)$ also is a solution of Eqs. (2.12). This will be useful below since asymptotic behavior of a solution typically involves the solution asymptoting towards a diagonal metric like for example the metric of Minkowski-space. The results here can also be used in a broader context to find corrections to solutions.

Now, Eqs. (2.12) for the perturbation $\delta G(r, z)$ becomes

$$
\vec{\nabla}^{2} \delta G_{i j}=\left(\frac{\vec{\nabla} G_{i i}}{G_{i i}}+\frac{\vec{\nabla} G_{j j}}{G_{j j}}\right) \cdot \vec{\nabla} \delta G_{i j}-\frac{\vec{\nabla} G_{i i}}{G_{i i}} \cdot \frac{\vec{\nabla} G_{j j}}{G_{j j}} \delta G_{i j} .
$$

We see here that the equations for $\delta G_{i j}$ are completely decoupled. I.e. we can solve for each component of $\delta G(r, z)$ separately. The only constraint is that $|\operatorname{det}(G+\delta G)|=r^{2}$. Using that $|\operatorname{det} G|=r^{2}$ this constraint can be written as $\operatorname{tr}\left(G^{-1} \delta G\right)=0$ which we again can write as

$$
\sum_{i=1}^{D-2} \frac{\delta G_{i i}}{G_{i i}}=0
$$

We see thus that only the diagonal components of $\delta G_{i i}$ are subject to a constraint. We note that for the diagonal components of $\delta G(r, z)$ the equations (4.1) can be written

$$
\vec{\nabla}^{2}\left(\frac{\delta G_{i i}}{G_{i i}}\right)=0
$$

for $i=1, \ldots, D-2$.

\footnotetext{
${ }^{9}$ Strictly speaking, one can consider higher-dimensional Minkowski-spaces $\mathcal{M}^{D}$ with $D \geq 6$, for example by making the split up $\mathcal{M}^{D}=\mathcal{M}^{4} \times \mathbb{R}^{D-4}$, with the $\mathbb{R}^{D-4}$ part spanned by the Killing vector fields. However, one can not use that to write any non-trivial solutions which asymptotes to $\mathcal{M}^{D}$, since any solution would be independent of the $\mathbb{R}^{D-4}$ part. Instead, one should consider $\mathcal{M}^{4} \times T^{D-4}$ or $\mathcal{M}^{5} \times T^{D-5}$.
} 


\subsection{Four-dimensional asymptotic Minkowski-space}

We consider in this section the four-dimensional Minkowski-space $\mathcal{M}^{4}$ and the asymptotic structure of solutions asymptoting to $\mathcal{M}^{4}$.

We first describe $D=4$ Minkowski-space $\mathcal{M}^{4}$. In terms of $G(r, z)$ we have that $\mathcal{M}^{4}$ is given by

$$
G_{11}=-1, G_{22}=r^{2},
$$

Thus, we have an infinite space-like $\operatorname{rod}[-\infty, \infty]$. In accordance with (2.13) we choose $e^{2 \nu}=1$. Demanding regularity of the solution near $r=0$ we get using (3.10) that $x^{2}=\phi$ should have period $2 \pi$. Making the coordinate transformation

$$
r=\rho \sin \theta, \quad z=\rho \cos \theta,
$$

we get the metric in spherical coordinates

$$
d s^{2}=-d t^{2}+\rho^{2} \sin ^{2} \theta d \phi^{2}+d \rho^{2}+\rho^{2} d \theta^{2},
$$

where we put $x^{1}=t$ and $x^{2}=\phi$.

If we consider a $D=4$ asymptotically Minkowski-space solution we have for $\rho \rightarrow \infty$ the corrections to the metric

$$
g_{t t}=-1+\frac{2 M}{\rho}+\mathcal{O}\left(\rho^{-2}\right), \quad g_{t \phi}=-2 J \frac{\sin ^{2} \theta}{\rho}\left(1+\mathcal{O}\left(\rho^{-1}\right)\right),
$$

with $g_{\phi \phi}=\rho^{2} \sin ^{2} \theta\left(1+\mathcal{O}\left(\rho^{-1}\right)\right)$.

In the $(r, z)$ canonical coordinates the asymptotic region corresponds to $\sqrt{r^{2}+z^{2}} \rightarrow \infty$ with $z / \sqrt{r^{2}+z^{2}}$ finite. In the canonical coordinates we have therefore from (4.7) the asymptotic behavior

$$
\begin{gathered}
G_{11}=-1+\frac{2 M}{\sqrt{r^{2}+z^{2}}}+\mathcal{O}\left(\left(r^{2}+z^{2}\right)^{-1}\right), \quad G_{12}=-\frac{2 J r^{2}}{\left(r^{2}+z^{2}\right)^{\frac{3}{2}}}+\mathcal{O}\left(\left(r^{2}+z^{2}\right)^{-1}\right), \\
G_{22}=r^{2}\left[1+\frac{2 M}{\sqrt{r^{2}+z^{2}}}+\mathcal{O}\left(\left(r^{2}+z^{2}\right)^{-1}\right)\right]
\end{gathered}
$$

for $\sqrt{r^{2}+z^{2}} \rightarrow \infty$ with $z / \sqrt{r^{2}+z^{2}}$ finite. For $G_{22}$ we used (4.2) and (4.3) of Section 4.1] We see from (4.8) that the leading asymptotic behavior of $G(r, z)$ is determined completely from $M$ and $J$. For $e^{2 \nu}$, the asymptotic behavior is simply that $e^{2 \nu} \simeq 1$ for $\sqrt{r^{2}+z^{2}} \rightarrow \infty$ with $z / \sqrt{r^{2}+z^{2}}$ finite.

\section{The period of $\phi$}

In the above we considered the period of $x^{2}=\phi$ to be $2 \pi$. We can also consider the more general case where $x^{2}=\phi$ has period $\Delta \phi=2 \pi \varepsilon$. Then the asymptotic behavior of a solution is

$$
G_{11} \simeq-1+\frac{2 M}{\varepsilon} \frac{1}{\sqrt{r^{2}+z^{2}}}, \quad G_{12} \simeq-\frac{2 J}{\varepsilon^{2}} \frac{r^{2}}{\left(r^{2}+z^{2}\right)^{\frac{3}{2}}}, \quad e^{2 \nu} \simeq \varepsilon^{2},
$$

for $\sqrt{r^{2}+z^{2}} \rightarrow \infty$ with $z / \sqrt{r^{2}+z^{2}}$ finite, where we used here a less precise notation than above for the sake of brevity. 


\subsection{Five-dimensional asymptotic Minkowski-space}

We consider in this section the five-dimensional Minkowski-space $\mathcal{M}^{5}$ and the asymptotic structure of solutions asymptoting to $\mathcal{M}^{5}$.

We first describe $D=5$ Minkowski-space $\mathcal{M}^{5}$. In terms of $G(r, z)$ we have that $\mathcal{M}^{5}$ is described by

$$
G_{11}=-1, \quad G_{22}=\sqrt{r^{2}+z^{2}}-z, G_{33}=\sqrt{r^{2}+z^{2}}+z .
$$

This corresponds to two semi-infinite rods $[-\infty, 0]$ and $[0, \infty]$. In accordance with Eqs. (2.13) we choose

$$
e^{2 \nu}=\frac{1}{2 \sqrt{r^{2}+z^{2}}} .
$$

Demanding regularity of the solution near $r=0$ we get using (3.10) that both $x^{2}$ and $x^{3}$ are periodic with period $2 \pi$. Making the coordinate transformation

$$
r=\frac{1}{2} \rho^{2} \sin 2 \theta, \quad z=\frac{1}{2} \rho^{2} \cos 2 \theta,
$$

we get the metric in spheroidal coordinates

$$
d s^{2}=-d t^{2}+\rho^{2} \sin ^{2} \theta d \phi^{2}+\rho^{2} \cos ^{2} \theta d \psi^{2}+d \rho^{2}+\rho^{2} d \theta^{2},
$$

where we put $x^{1}=t, x^{2}=\phi$ and $x^{3}=\psi$. We remind the reader that regularity of the solution requires both $x^{2}=\phi$ and $x^{3}=\psi$ to be periodic with period $2 \pi$.

If we consider a $D=5$ asymptotically Minkowski-space solution we have for $\rho \rightarrow \infty$ the corrections to the metric

$$
\begin{gathered}
g_{t t}=-1+\frac{8 M}{3 \pi} \frac{1}{\rho^{2}}+\mathcal{O}\left(\rho^{-4}\right) \\
g_{t \phi}=-\frac{4 J_{1}}{\pi} \frac{\sin ^{2} \theta}{\rho^{2}}\left(1+\mathcal{O}\left(\rho^{-2}\right)\right), \quad g_{t \psi}=-\frac{4 J_{2}}{\pi} \frac{\cos ^{2} \theta}{\rho^{2}}\left(1+\mathcal{O}\left(\rho^{-2}\right)\right),
\end{gathered}
$$

with $g_{\phi \phi}=\rho^{2} \sin ^{2} \theta\left(1+\mathcal{O}\left(\rho^{-2}\right)\right)$ and $g_{\psi \psi}=\rho^{2} \cos ^{2} \theta\left(1+\mathcal{O}\left(\rho^{-2}\right)\right)$. Using this together with (4.1) and (4.2), we get the asymptotics in the $(r, z)$ canonical coordinates

$$
\begin{gathered}
G_{11}=-1+\frac{4 M}{3 \pi} \frac{1}{\sqrt{r^{2}+z^{2}}}+\mathcal{O}\left(\left(r^{2}+z^{2}\right)^{-1}\right), \quad G_{23}=\zeta \frac{r^{2}}{\left(r^{2}+z^{2}\right)^{\frac{3}{2}}}+\mathcal{O}\left(\left(r^{2}+z^{2}\right)^{-1}\right), \\
G_{12}=-\frac{J_{1}}{\pi} \frac{\sqrt{r^{2}+z^{2}}-z}{r^{2}+z^{2}}+\mathcal{O}\left(\left(r^{2}+z^{2}\right)^{-1}\right), G_{13}=-\frac{J_{2}}{\pi} \frac{\sqrt{r^{2}+z^{2}}+z}{r^{2}+z^{2}}+\mathcal{O}\left(\left(r^{2}+z^{2}\right)^{-1}\right), \\
G_{22}=\left(\sqrt{r^{2}+z^{2}}-z\right)\left[1+\frac{2}{3 \pi} \frac{M+\eta}{\sqrt{r^{2}+z^{2}}}+\mathcal{O}\left(\left(r^{2}+z^{2}\right)^{-1}\right)\right], \\
G_{33}=\left(\sqrt{r^{2}+z^{2}}+z\right)\left[1+\frac{2}{3 \pi} \frac{M-\eta}{\sqrt{r^{2}+z^{2}}}+\mathcal{O}\left(\left(r^{2}+z^{2}\right)^{-1}\right)\right],
\end{gathered}
$$

for $\sqrt{r^{2}+z^{2}} \rightarrow \infty$ with $z / \sqrt{r^{2}+z^{2}}$ finite, where $\zeta$ and $\eta$ are constants. Note that $\eta$ changes under the transformation $z \rightarrow z+$ constant and is thus not a gauge-invariant parameter, unlike $\zeta$. Finally, we remark that the asymptotics of $e^{2 \nu}$ is

$$
e^{2 \nu} \simeq \frac{1}{2 \sqrt{r^{2}+z^{2}}},
$$


for $\sqrt{r^{2}+z^{2}} \rightarrow \infty$ with $z / \sqrt{r^{2}+z^{2}}$ finite.

\section{The periods of $\phi$ and $\psi$}

The periods of $x^{2}=\phi$ and $x^{3}=\psi$ were chosen to be $2 \pi$ in the above. We consider here the more general case where the period $\Delta \phi$ of $x^{2}=\phi$ and the period $\Delta \psi$ of $x^{3}=\psi$ are given by $\Delta \phi=\Delta \psi=2 \pi \varepsilon$. Then the asymptotics of $G_{i j}(r, z)$ and $e^{2 \nu}$ takes the form

$$
\begin{gathered}
G_{11} \simeq-1+\frac{4 M}{3 \pi \varepsilon^{2}} \frac{1}{\sqrt{r^{2}+z^{2}}}, \quad G_{23} \simeq \frac{\zeta}{\varepsilon^{4}} \frac{r^{2}}{\left(r^{2}+z^{2}\right)^{\frac{3}{2}}}, \quad e^{2 \nu} \simeq \frac{\varepsilon^{2}}{2 \sqrt{r^{2}+z^{2}}}, \\
G_{12} \simeq-\frac{J_{1}}{\pi \varepsilon^{3}} \frac{\sqrt{r^{2}+z^{2}}-z}{r^{2}+z^{2}}, \quad G_{22} \simeq\left(\sqrt{r^{2}+z^{2}}-z\right)\left[1+\frac{2}{3 \pi \varepsilon^{2}} \frac{M+\eta}{\sqrt{r^{2}+z^{2}}}\right], \\
G_{13} \simeq-\frac{J_{2}}{\pi \varepsilon^{3}} \frac{\sqrt{r^{2}+z^{2}}+z}{r^{2}+z^{2}}, \quad G_{33} \simeq\left(\sqrt{r^{2}+z^{2}}+z\right)\left[1+\frac{2}{3 \pi \varepsilon^{2}} \frac{M-\eta}{\sqrt{r^{2}+z^{2}}}\right],
\end{gathered}
$$

for $\sqrt{r^{2}+z^{2}} \rightarrow \infty$ with $z / \sqrt{r^{2}+z^{2}}$ finite, where we used here a less precise notation than above for the sake of brevity.

\section{Rotating black hole solutions}

In this section we consider rotating black hole solutions in four and five dimensions and describe them using the canonical form of the metric (2.10)-(2.11).

\subsection{Kerr solution}

We first consider the four-dimensional Kerr solution [1] which corresponds to a rotating black hole. The topology of the event horizon is that of a two-sphere $S^{2}$. It is already known how to write the Kerr solution in the canonical form (2.10)-(2.11) (see for example [12, 14, 15]), but we review this here for completeness, and since it illustrates the methods developed in Section 3 .

The Kerr metric in Boyer-Linquist coordinates is

$$
\begin{aligned}
d s^{2}= & -\frac{\Delta-a^{2} \sin ^{2} \theta}{\Sigma} d t^{2}-2 a \sin ^{2} \theta \frac{\rho^{2}+a^{2}-\Delta}{\Sigma} d t d \phi \\
& +\frac{\left(\rho^{2}+a^{2}\right)^{2}-\Delta a^{2} \sin ^{2} \theta}{\Sigma} \sin ^{2} \theta d \phi^{2}+\frac{\Sigma}{\Delta} d \rho^{2}+\Sigma d \theta^{2}
\end{aligned}
$$

with

$$
\Delta=\rho^{2}-2 M \rho+a^{2}, \quad \Sigma=\rho^{2}+a^{2} \cos ^{2} \theta .
$$

The coordinates for the two Killing directions are $x^{1}=t$ and $x^{2}=\phi$. From $\operatorname{det}(G)=$ $-\Delta \sin ^{2} \theta$ we get the $r$-coordinate, and it is a straightforward exercise to find a $z$-coordinate so that the metric fits into the ansatz (2.10). We find

$$
r=\sqrt{\Delta} \sin \theta, \quad z=(\rho-M) \cos \theta .
$$


Using this, we can in principle write the Kerr metric in the canonical form (2.10)-(2.11) . However, it is useful to instead first write the Kerr-metric in the prolate spherical coordinates (see Appendix G). From the definition (G.1) of the prolate spherical coordinates $(x, y)$ we see that

$$
\alpha^{2}\left(x^{2}-1\right)\left(1-y^{2}\right)=\Delta \sin ^{2} \theta, \quad \alpha x y=(\rho-M) \cos \theta,
$$

Using the ansatz $x=x(\rho)$ and $y=y(\theta)$ we get

$$
x=\frac{\rho-M}{\sqrt{M^{2}-a^{2}}}, \quad y=\cos \theta, \quad \alpha=\sqrt{M^{2}-a^{2}} .
$$

We compute

$$
\begin{gathered}
G_{11}=-\frac{x^{2} \cos ^{2} \lambda+y^{2} \sin ^{2} \lambda-1}{(1+x \cos \lambda)^{2}+y^{2} \sin ^{2} \lambda}, \quad G_{12}=-2 a \frac{\left(1-y^{2}\right)(1+x \cos \lambda)}{(1+x \cos \lambda)^{2}+y^{2} \sin ^{2} \lambda}, \\
e^{2 \nu}=\frac{(1+x \cos \lambda)^{2}+y^{2} \sin ^{2} \lambda}{\left(x^{2}-y^{2}\right) \cos ^{2} \lambda}
\end{gathered}
$$

where we defined

$$
\sin \lambda=\frac{a}{M}
$$

The $G_{22}$ component can be found from

$$
G_{22}=\frac{G_{12}^{2}-\alpha^{2}\left(x^{2}-1\right)\left(1-y^{2}\right)}{G_{11}} .
$$

We obtained now $G_{i j}$ and $e^{2 \nu}$ as functions of $x$ and $y$. From this it is straightforward to use Eq. (G.7) to get $G_{i j}$ and $e^{2 \nu}$ as functions of $r$ and $z$.

\section{Asymptotic region}

Using (G.9) we find that in the asymptotic region $\sqrt{r^{2}+z^{2}} \rightarrow \infty$ with $z / \sqrt{r^{2}+z^{2}}$ finite, we have

$$
G_{11}=-1+\frac{2 M}{\sqrt{r^{2}+z^{2}}}+\mathcal{O}\left(\left(r^{2}+z^{2}\right)^{-1}\right), \quad G_{12}=-2 M a \frac{r^{2}}{\left(r^{2}+z^{2}\right)^{3 / 2}}+\mathcal{O}\left(\left(r^{2}+z^{2}\right)^{-1}\right) .
$$

From (4.8) we see that this means that $M$ is the mass, which justifies our use of this symbol in the solution, and that the angular momentum is $J=M a$.

Note that $e^{2 \nu} \simeq 1$ in the asymptotic region. From Section 4.2 we know that this means that $\Delta \phi=2 \pi$, i.e. that $\phi$ is required to have period $2 \pi$. This can also be found directly from the solution near $r=0$ using the analysis of Section 3.1

\section{Rod-structure}

We now analyse the rod-structure of the Kerr solution according to the methods of Section 3. We have: 
- The two semi-infinite space-like rods $[-\infty,-\alpha]$ and $[\alpha, \infty]$. For $z \in[-\infty,-\alpha]$ and $r=0$ we see from (G.7) that $x=-z / \alpha$ and $y=-1$. Similarly, for $z \in[\alpha, \infty]$ and $r=0$ we have that $x=z / \alpha$ and $y=1$. Considering Eqs. (5.6) and (5.8) we see that for both intervals $G_{12}=G_{22}=0$ while $G_{11} \neq 0$. By Eq. (3.8) this means that the two rods both are in the direction $v=(0,1)$, i.e. that the rods are in the $\partial / \partial x^{2}$ direction and therefore space-like.

- The finite time-like $\operatorname{rod}[-\alpha, \alpha]$. For $z \in[-\alpha, \alpha]$ we see from (G.7) that $x=1$ and $y=z / \alpha$. Considering Eqs. (5.6) and (5.8) we see that $\sum_{j=1}^{2} G_{i j} v^{j}=0$ for $z \in[-\alpha, \alpha]$ with

$$
v=(1, \Omega), \quad \Omega=\frac{\sin \lambda}{2 M(1+\cos \lambda)} .
$$

This means that we have a rod $[-\alpha, \alpha]$ along the direction (5.10). Since $G_{i j} v^{i} v^{j} / r^{2}$ is negative for $r \rightarrow 0$ the rod is time-like. Note that $\Omega$ in (5.10) is the angular velocity of the event horizon. ${ }^{10}$ One finds easily that this rod corresponds to an event horizon of topology $S^{2}$. This is a consequence of the fact that the rods on each side of the $[-\alpha, \alpha]$ rod are in the same space-like direction, i.e. the $\partial / \partial x^{2}$ direction.

For the time-like rod $[-\alpha, \alpha]$ we see from (15.10) that if we change coordinates as $\tilde{x}^{1}=x^{1}$ and $\tilde{x}^{2}=x^{2}-\Omega x^{1}$, then in these coordinates the $[-\alpha, \alpha]$ rod is along the $\partial / \partial \tilde{x}^{1}$ direction. This means that $\tilde{x}^{1}$ and $\tilde{x}^{2}$ are two of the coordinates of the comoving coordinates for the Kerr solution since the comoving coordinates precisely gives a diagonal metric at the horizon. In other words, finding the direction of the $[-\alpha, \alpha]$ rod precisely corresponds to finding the comoving coordinates near the horizon.

Finally, in the accordance with the ideas of Section 3.2, we note that we can make an alternative parameterization of the Kerr solution by stating that we have three rods, the two rods $[-\infty,-\alpha]$ and $[\alpha, \infty]$ in the $x^{2}$ direction and the $\operatorname{rod}[-\alpha, \alpha]$ in the $(1, \Omega)$ direction. Then the whole Kerr solution can be parameterized uniquely by the two parameters $\alpha$ and $\Omega$.

\subsection{Five-dimensional Myers-Perry solution}

The five-dimensional Myers-Perry solution [7] corresponds to a five-dimensional spinning black hole. ${ }^{11}$ This is an asymptotically flat stationary solution of the vacuum Einstein equations with an event horizon that has the topology of a three-sphere $S^{3}$.

\footnotetext{
${ }^{10}$ Note that $v$ in (5.10) precisely is the null Killing vector for the Killing horizon (the event horizon), since $v=\sum_{i=1}^{2} v^{i} V_{(i)}$, and since $v^{2}=G_{i j} v^{i} v^{j}=0$ for $r=0$ and $z \in[-\alpha, \alpha]$. In other words, for a Killing horizon the null Killing vector is the same as the direction of the time-like rod.

${ }^{11}$ The five-dimensional Myers-Perry black hole generalizes the static Schwarzschild-Tangherlini black hole [19].
} 
The metric of the five-dimensional Myers-Perry black hole is

$$
\begin{aligned}
d s^{2}= & -d t^{2}+\frac{\rho_{0}^{2}}{\Sigma}\left[d t-a_{1} \sin ^{2} \theta d \phi-a_{2} \cos ^{2} \theta d \psi\right]^{2} \\
& +\left(\rho^{2}+a_{1}^{2}\right) \sin ^{2} \theta d \phi^{2}+\left(\rho^{2}+a_{2}^{2}\right) \cos ^{2} \theta d \psi^{2}+\frac{\Sigma}{\Delta} d \rho^{2}+\Sigma d \theta^{2},
\end{aligned}
$$

where

$$
\Delta=\rho^{2}\left(1+\frac{a_{1}^{2}}{\rho^{2}}\right)\left(1+\frac{a_{2}^{2}}{\rho^{2}}\right)-\rho_{0}^{2}, \quad \Sigma=\rho^{2}+a_{1}^{2} \cos ^{2} \theta+a_{2}^{2} \sin ^{2} \theta .
$$

The coordinates for the three Killing directions are $x^{1}=t, x^{2}=\phi$ and $x^{3}=\psi$. We now transform this metric to the canonical form (2.10)-(2.11). We compute that $\operatorname{det} G=$ $-\frac{1}{4} \rho^{2} \Delta \sin ^{2} 2 \theta$. From this we can determine $r$, and $z$ can be found by demanding the metric to be of the form (2.10). We get

$$
r=\frac{1}{2} \rho \sqrt{\Delta} \sin 2 \theta, \quad z=\frac{1}{2} \rho^{2}\left(1-\frac{\rho_{0}^{2}-a_{1}^{2}-a_{2}^{2}}{2 \rho^{2}}\right) \cos 2 \theta .
$$

This determines in principle how the Myers-Perry metric (5.11) should transform to the form (2.10). However, as for the Kerr metric, it is convinient to express the MyersPerry metric in prolate spherical coordinates, defined in Appendix G. instead. From the definition of the prolate spherical coordinates (G.1) we see that

$$
\alpha^{2}\left(x^{2}-1\right)\left(1-y^{2}\right)=\frac{1}{4} \rho^{2} \Delta \sin ^{2} 2 \theta, \quad \alpha x y=\frac{1}{2} \rho^{2}\left(1-\frac{\rho_{0}^{2}-a_{1}^{2}-a_{2}^{2}}{2 \rho^{2}}\right) \cos 2 \theta .
$$

If we try the ansatz $x=x(\rho)$ and $y=y(\theta)$ we get

$$
x=\frac{2 \rho^{2}+a_{1}^{2}+a_{2}^{2}-\rho_{0}^{2}}{\sqrt{\left(\rho_{0}^{2}-a_{1}^{2}-a_{2}^{2}\right)^{2}-4 a_{1}^{2} a_{2}^{2}}}, \quad y=\cos 2 \theta, \quad \alpha=\frac{1}{4} \sqrt{\left(\rho_{0}^{2}-a_{1}^{2}-a_{2}^{2}\right)^{2}-4 a_{1}^{2} a_{2}^{2}} .
$$

Using this, we can write $G_{i j}$ and $e^{2 \nu}$ in terms of the prolate spherical coordinates. We get

$$
\begin{gathered}
G_{11}=-\frac{4 \alpha x+\left(a_{1}^{2}-a_{2}^{2}\right) y-\rho_{0}^{2}}{4 \alpha x+\left(a_{1}^{2}-a_{2}^{2}\right) y+\rho_{0}^{2}}, \quad G_{12}=-\frac{a_{1} \rho_{0}^{2}(1-y)}{4 \alpha x+\left(a_{1}^{2}-a_{2}^{2}\right) y+\rho_{0}^{2}}, \\
G_{13}=-\frac{a_{2} \rho_{0}^{2}(1+y)}{4 \alpha x+\left(a_{1}^{2}-a_{2}^{2}\right) y+\rho_{0}^{2}}, \quad G_{23}=\frac{1}{2} \frac{a_{1} a_{2} \rho_{0}^{2}\left(1-y^{2}\right)}{4 \alpha x+\left(a_{1}^{2}-a_{2}^{2}\right) y+\rho_{0}^{2}}, \\
G_{22}=\frac{1-y}{4}\left[4 \alpha x+\rho_{0}^{2}+a_{1}^{2}-a_{2}^{2}+\frac{2 a_{1}^{2} \rho_{0}^{2}(1-y)}{4 \alpha x+\left(a_{1}^{2}-a_{2}^{2}\right) y+\rho_{0}^{2}}\right], \\
G_{33}=\frac{1+y}{4}\left[4 \alpha x+\rho_{0}^{2}-a_{1}^{2}+a_{2}^{2}+\frac{2 a_{2}^{2} \rho_{0}^{2}(1+y)}{4 \alpha x+\left(a_{1}^{2}-a_{2}^{2}\right) y+\rho_{0}^{2}}\right], \\
e^{2 \nu}=\frac{4 \alpha x+\left(a_{1}^{2}-a_{2}^{2}\right) y+\rho_{0}^{2}}{8 \alpha^{2}\left(x^{2}-y^{2}\right)} .
\end{gathered}
$$

Using Eq. (G.7) it is now a straightforward exercise to write the components $G_{i j}$ and $e^{2 \nu}$ as functions of the canonical $(r, z)$ coordinates. 


\section{Asymptotic region}

Regarding (5.16) as functions of the canonical coordinates $(r, z)$, we find that $G_{i j}(r, z)$ in the asymptotic region, $\sqrt{r^{2}+z^{2}} \rightarrow \infty$ with $z / \sqrt{r^{2}+z^{2}}$ finite, behaves as

$$
\begin{gathered}
G_{11}=-1+\frac{\rho_{0}^{2}}{2 \sqrt{r^{2}+z^{2}}}+\mathcal{O}\left(\left(r^{2}+z^{2}\right)^{-1}\right), G_{12}=-\frac{a_{1} \rho_{0}^{2}}{4} \frac{\sqrt{r^{2}+z^{2}}-z}{r^{2}+z^{2}}+\mathcal{O}\left(\left(r^{2}+z^{2}\right)^{-1}\right), \\
G_{13}=-\frac{a_{2} \rho_{0}^{2}}{4} \frac{\sqrt{r^{2}+z^{2}}+z}{r^{2}+z^{2}}+\mathcal{O}\left(\left(r^{2}+z^{2}\right)^{-1}\right), \quad G_{23}=\frac{a_{1} a_{2} \rho_{0}^{2} r^{2}}{8\left(r^{2}+z^{2}\right)^{3 / 2}}+\mathcal{O}\left(\left(r^{2}+z^{2}\right)^{-1}\right), \\
G_{22}=\left(\sqrt{r^{2}+z^{2}}-z\right)\left[1+\frac{\rho_{0}^{2}+a_{1}^{2}-a_{2}^{2}}{4 \sqrt{r^{2}+z^{2}}}+\mathcal{O}\left(\left(r^{2}+z^{2}\right)^{-1}\right)\right], \\
G_{33}=\left(\sqrt{r^{2}+z^{2}}+z\right)\left[1+\frac{\rho_{0}^{2}-a_{1}^{2}+a_{2}^{2}}{4 \sqrt{r^{2}+z^{2}}}+\mathcal{O}\left(\left(r^{2}+z^{2}\right)^{-1}\right)\right] .
\end{gathered}
$$

We can now use (4.15) to read off the asymptotic quantities. We get

$$
M=\frac{3 \pi}{8} \rho_{0}^{2}, \quad J_{1}=\frac{\pi}{4} a_{1} \rho_{0}^{2}, \quad J_{2}=\frac{\pi}{4} a_{2} \rho_{0}^{2}, \quad \zeta=\frac{1}{8} a_{1} a_{2} \rho_{0}^{2}, \quad \eta=\frac{3 \pi}{8}\left(a_{1}^{2}-a_{2}^{2}\right) .
$$

Note that one can see from the above results that $e^{2 \nu} \simeq 1 /\left(2 \sqrt{r^{2}+z^{2}}\right)$. From Section 4.3 we have that this means $x^{2}=\phi$ and $x^{3}=\psi$ are periodic with period $2 \pi$.

\section{Rod-structure}

We now analyse the rod-structure of the five-dimensional Myers-Perry solution according to the methods of Section 3. We have

- The semi-infinite space-like $\operatorname{rod}[-\infty,-\alpha]$. For $z \in[-\infty,-\alpha]$ and $r=0$ we see from (G.7) that $x=-z / \alpha$ and $y=-1$. From (5.16) we see then that $G_{13}=G_{23}=G_{33}=$ 0. By Eq. (3.8) we see that this rod has the direction $v=(0,0,1)$, i.e. it is in the $\partial / \partial x^{3}$ direction.

- The finite time-like rod $[-\alpha, \alpha]$. For $z \in[-\alpha, \alpha]$ we see from (G.7) that $x=1$ and $y=z / \alpha$. Using (15.16), we see that $\sum_{j=1}^{3} G_{i j} v^{j}=0$ for $z \in[-\alpha, \alpha]$ with $v$ being the vector

$$
v=\left(1, \Omega_{1}, \Omega_{2}\right),
$$

with

$$
\Omega_{1}=\frac{\rho_{0}^{2}+a_{1}^{2}-a_{2}^{2}-4 \alpha}{2 a_{1} \rho_{0}^{2}}, \quad \Omega_{2}=\frac{\rho_{0}^{2}-a_{1}^{2}+a_{2}^{2}-4 \alpha}{2 a_{2} \rho_{0}^{2}} .
$$

Therefore, the $\operatorname{rod}[-\alpha, \alpha]$ is in the direction $v$ given by (5.19). Note that $\Omega_{1}$ and $\Omega_{2}$ are the angular velocities of the Myers-Perry black hole. That the $\operatorname{rod}[-\alpha, \alpha]$ is time-like can be seen by noting that $G_{i j} v^{i} v^{j} / r^{2}$ is negative for $r \rightarrow 0$. One can check that this rod corresponds to an event horizon with topology $S^{3}$. This follows from the fact that the $[-\alpha, \alpha]$ rod has space-like rods on each side in two different directions. 
- The semi-infinite space-like $\operatorname{rod}[\alpha, \infty]$. For $z \in[\alpha, \infty]$ and $r=0$ we see that $x=z / \alpha$ and $y=1$. From (5.16) we see then that $G_{12}=G_{22}=G_{23}=0$. This means that the $\operatorname{rod}[\alpha, \infty]$ is in the direction $v=(0,1,0)$, i.e. in the $\partial / \partial x^{2}$ direction.

We see that one can use the direction (5.19) to transform to coordinates $\left(\tilde{x}^{1}, \tilde{x}^{2}, \tilde{x}^{3}\right)=$ $\left(x^{1}, x^{2}-\Omega_{1} x^{1}, x^{3}-\Omega_{2} x^{1}\right)$ so that the $[-\alpha, \alpha] \operatorname{rod}$ is along the $\partial / \partial \tilde{x}^{1}$ direction. This means that $\left(\tilde{x}^{1}, \tilde{x}^{2}, \tilde{x}^{3}\right)$ are comoving coordinates for the event horizon. Thus, finding the direction of the rod $[-\alpha, \alpha]$ corresponds to finding the comoving coordinates on the horizon of the Myers-Perry black hole.

We note furthermore that we can make an alternative parameterization of the fivedimensional Myers-Perry solution. Clearly, the direction $v=\left(1, \Omega_{1}, \Omega_{2}\right)$ in (5.19) is given uniquely by the two parameters $\Omega_{1}$ and $\Omega_{2}$. Letting then $\alpha$ be the third parameter, we have that the five-dimensional Myers-Perry solution is characterized uniquely by the three parameters $\alpha, \Omega_{1}$ and $\Omega_{2}$, in accordance with the ideas of Section 3.2

\subsection{Myers-Perry solution with one angular momentum}

In the following we give details on the five-dimensional Myers-Perry solution with one angular momentum. In our conventions, we obtain the Myers-Perry solution with one angular momentum below from the Myers-Perry solution with two angular momenta above by setting $a_{1}=a$ and $a_{2}=0$. The metric is

$$
\begin{aligned}
d s^{2}= & -d t^{2}+\frac{\rho_{0}^{2}}{\Sigma}\left(d t-a \sin ^{2} \theta d \phi\right)^{2}+\left(\rho^{2}+a^{2}\right) \sin ^{2} \theta d \phi^{2}+\rho^{2} \cos ^{2} \theta d \psi^{2} \\
& +\frac{\Sigma}{\Delta} d \rho^{2}+\Sigma d \theta^{2}
\end{aligned}
$$

where

$$
\Delta=\rho^{2}-\rho_{0}^{2}+a^{2}, \quad \Sigma=\rho^{2}+a^{2} \cos ^{2} \theta .
$$

From (5.13) we see that the $(r, z)$ coordinates are given by

$$
r=\frac{1}{2} \rho \sqrt{\Delta} \sin 2 \theta, \quad z=\left(\frac{1}{2} \rho^{2}-\frac{\rho_{0}^{2}-a^{2}}{4}\right) \cos 2 \theta .
$$

The prolate spherical coordinates are given by

$$
x=\frac{2 \rho^{2}}{\rho_{0}^{2}-a^{2}}-1, \quad y=\cos 2 \theta, \quad \alpha=\frac{\rho_{0}^{2}-a^{2}}{4},
$$

as one can see from (5.15). From Eqs. (5.16) we obtain that the metric in prolate spherical coordinates is given by

$$
\begin{gathered}
G_{11}=-\frac{x \cos ^{2} \lambda+y \sin ^{2} \lambda-1}{x \cos ^{2} \lambda+y \sin ^{2} \lambda+1}, \quad G_{12}=-\frac{2 \sqrt{\alpha} \tan \lambda(1-y)}{x \cos ^{2} \lambda+y \sin ^{2} \lambda+1}, \\
G_{22}=\frac{\alpha}{\cos ^{2} \lambda}(1-y)\left[x \cos ^{2} \lambda+1+\sin ^{2} \lambda+\frac{2 \sin ^{2} \lambda(1-y)}{x \cos ^{2} \lambda+y \sin ^{2} \lambda+1}\right], \\
G_{33}=\alpha(x+1)(1+y), \quad e^{2 \nu}=\frac{x \cos ^{2} \lambda+y \sin ^{2} \lambda+1}{2 \alpha \cos ^{2} \lambda\left(x^{2}-y^{2}\right)}
\end{gathered}
$$


where we have defined

$$
\sin \lambda=\frac{a}{\rho_{0}} .
$$

Using Eq. (G.7) we get furthermore the metric written in the canonical form (2.10)-(2.11) as functions of the canonical $(r, z)$ coordinates

$$
\begin{gathered}
G_{11}=-\frac{R_{+}+R_{-} \cos 2 \lambda-2 \alpha}{R_{+}+R_{-} \cos 2 \lambda+2 \alpha}, \quad G_{12}=-\frac{2 \sqrt{\alpha} \tan \lambda\left(2 \alpha-R_{+}+R_{-}\right)}{R_{+}+R_{-} \cos 2 \lambda+2 \alpha}, \\
G_{22}=\frac{2 \alpha-R_{+}+R_{-}}{4 \alpha}\left[R_{+}+R_{-}+2 \alpha \frac{1+\sin ^{2} \lambda}{\cos ^{2} \lambda}+\frac{4 \alpha \tan ^{2} \lambda\left(2 \alpha-R_{+}+R_{-}\right)}{R_{+}+R_{-} \cos 2 \lambda+2 \alpha}\right], \\
G_{33}=R_{+}+z+\alpha, \quad e^{2 \nu}=\frac{R_{+}+R_{-} \cos 2 \lambda+2 \alpha}{4 R_{+} R_{-} \cos ^{2} \lambda},
\end{gathered}
$$

with

$$
R_{+}=\sqrt{r^{2}+(z+\alpha)^{2}}, \quad R_{-}=\sqrt{r^{2}+(z-\alpha)^{2}} .
$$

We see that the whole solution (5.27) is determined by the two parameters $\alpha$ and $\lambda$.

From the analysis of the asymptotic region of the Myers-Perry solution with two angular momenta, we see that the asymptotic quantities are now

$$
M=\frac{3 \pi}{8} \rho_{0}^{2}, \quad J_{1}=\frac{\pi}{4} a \rho_{0}^{2}, \quad J_{2}=0, \quad \zeta=0, \quad \eta=\frac{3 \pi}{8} a^{2} .
$$

We list for completeness here the rod-structure of the solution (5.27). This can easily be obtained using the results of the analysis for the case of two angular momenta.

- The semi-infinite space-like $\operatorname{rod}[-\infty,-\alpha]$. This rod is in the direction $v=(0,0,1)$, i.e. in the $\partial / \partial x^{3}$ direction.

- The finite time-like rod $[-\alpha, \alpha]$. This rod is in the direction $v$ given by

$$
v=(1, \Omega, 0), \quad \Omega=\frac{a}{\rho_{0}^{2}}=\frac{\sin \lambda \cos \lambda}{2 \sqrt{\alpha}} .
$$

- The semi-infinite space-like $\operatorname{rod}[\alpha, \infty]$. This $\operatorname{rod}$ is in the direction $v=(0,1,0)$, i.e. in the $\partial / \partial x^{2}$ direction.

\section{Black ring solutions}

In this section we consider the rotating black ring [6]. The rotating black ring is the first known example of a stationary and regular asymptotically flat five-dimensional solution with an event horizon that is not topologically a three-sphere $S^{3}$. Instead the horizon is topologically a ring $S^{2} \times S^{1}$.

We first describe in Section 6.1 the general black ring solution which generically has a conical singularity. We write its metric in the canonical form (2.10)-(2.11) and discuss the rod-structure. We consider then briefly the special case of the static black ring solution 
and furthermore how to obtain the Myers-Perry rotating black hole with one angular momentum.

In Section 6.2 we present the regular rotating black ring, write its metric in the canonical form (2.10)-(2.11) and discuss its properties.

\subsection{General black ring solution}

We begin by reviewing briefly the general black ring metric. So far, the general black ring metric has been written only in the so-called C-metric coordinates. In the C-metric coordinates of [20], the general black ring metric is ${ }^{12}$

$$
d s^{2}=-\frac{F(v)}{F(u)}\left(d t-C \kappa \frac{1+v}{F(v)} d \phi\right)^{2}+\frac{2 \kappa^{2} F(u)}{(u-v)^{2}}\left[-\frac{G(v)}{F(v)} d \phi^{2}+\frac{G(u)}{F(u)} d \psi^{2}+\frac{d u^{2}}{G(u)}-\frac{d v^{2}}{G(v)}\right] .
$$

Here $F(\xi)$ and $G(\xi)$ are the structure functions, which takes the form

$$
F(\xi)=1+b \xi, \quad G(\xi)=\left(1-\xi^{2}\right)(1+c \xi)
$$

where the parameters $b$ and $c$ lie in the ranges

$$
0<c \leq b<1
$$

Furthermore, in (6.1), the constant $C$ is given in terms of $b$ and $c$ by

$$
C=\sqrt{2 b(b-c) \frac{1+b}{1-b}} .
$$

The $u$ and $v$ coordinates in (6.1) have the ranges

$$
-1 \leq u \leq 1, \quad v \leq-1
$$

Note that the solution (6.1) generically has conical singularities at $u=1, u=-1$ and $v=-1$ [20]. These will be analyzed below using the methods of Section 3, We note here that while the potential singularities at $u=-1$ and $v=-1$ will be cured by choosing the periods of $x^{2}=\phi$ and $x^{3}=\psi$ appropiately, we do not fix the singularity at $u=1$ before in Section 6.2 where we consider the regular rotating black ring. Thus, in the following the black ring solution is generically singular at $u=1$.

\footnotetext{
${ }^{12}$ We use here the C-metric coordinates of [20], since they are particular convinient for our purposes. There have been given three different, but equivalent, C-metric coordinates for the black ring in the literature: i) The original coordinates of [6]. ii) The coordinates described in [21] where one takes the solution of [6] and rewrite it so that structure functions are factorizable. iii) The coordinates of [20] where the structure functions also are factorizable, but where $\operatorname{det} G$ is simpler.
} 


\section{Metric in canonical coordinates}

We now find the metric in the canonical coordinates (2.10)-(2.11). In the following we use extensively the results of Appendix $\mathrm{H}$ In Appendix $\mathrm{H}$ the general relation between C-metric coordinates $(u, v)$ and the canonical coordinates $(r, z)$ is discussed in detail. Furthermore, for the specific case relevant here several useful relations between the Cmetric coordinates $(u, v)$ and the canonical coordinates $(r, z)$ are given.

We take the coordinates for the Killing directions to be $x^{1}=t, x^{2}=\phi$ and $x^{3}=\psi$.

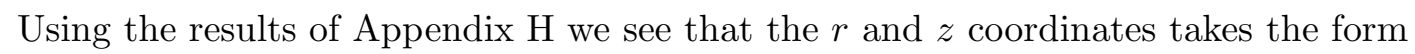

$$
r=\frac{2 \kappa^{2} \sqrt{-G(u) G(v)}}{(u-v)^{2}}, \quad z=\frac{\kappa^{2}(1-u v)(2+c u+c v)}{(u-v)^{2}} .
$$

This is obtained by first computing $\operatorname{det} G$, which gives $r$. In Appendix $\mathbf{H}$ it is then found for this particular $r$, given by the structure function $G(\xi)$ in (6.2), that $z$ can be chosen as in (H.12).

From (6.1) and (6.2) we get using (H.18) of Appendix $[\mathrm{H}$ giving $u$ and $v$ as functions of $r$ and $z$, that $G_{i j}(r, z)$ is

$$
\begin{gathered}
G_{11}=-\frac{(1+b)(1-c) R_{1}+(1-b)(1+c) R_{2}-2(b-c) R_{3}-2 b\left(1-c^{2}\right) \kappa^{2}}{(1+b)(1-c) R_{1}+(1-b)(1+c) R_{2}-2(b-c) R_{3}+2 b\left(1-c^{2}\right) \kappa^{2}}, \\
G_{12}=-\frac{2 C \kappa(1-c)\left(R_{3}-R_{1}+(1+c) \kappa^{2}\right)}{(1+b)(1-c) R_{1}+(1-b)(1+c) R_{2}-2(b-c) R_{3}+2 b\left(1-c^{2}\right) \kappa^{2}} \\
G_{33}=\frac{\left(R_{1}+R_{2}+2 c \kappa^{2}\right)\left(R_{1}-R_{3}+(1+c) \kappa^{2}\right)\left(R_{2}+R_{3}-(1-c) \kappa^{2}\right)}{2 \kappa^{2}\left((1-c) R_{1}-(1+c) R_{2}-2 c R_{3}\right)}
\end{gathered}
$$

where we have defined $R_{1}, R_{2}$ and $R_{3}$ by

$$
R_{1}=\sqrt{r^{2}+\left(z+c \kappa^{2}\right)^{2}}, \quad R_{2}=\sqrt{r^{2}+\left(z-c \kappa^{2}\right)^{2}}, \quad R_{3}=\sqrt{r^{2}+\left(z-\kappa^{2}\right)^{2}},
$$

as also defined in (H.15). For simplicity, we do not write $G_{22}$ explicitly here, but note that it is given implicitly as a function of $(r, z)$ by

$$
G_{22}=-\frac{r^{2}}{G_{11} G_{33}}+\frac{G_{12}^{2}}{G_{11}}
$$

Using now furthermore (H.10), we get

$$
\begin{aligned}
e^{2 \nu}= & {\left[(1+b)(1-c) R_{1}+(1-b)(1+c) R_{2}+2(c-b) R_{3}+2 b\left(1-c^{2}\right) \kappa^{2}\right] } \\
& \times \frac{(1-c) R_{1}+(1+c) R_{2}+2 c R_{3}}{8\left(1-c^{2}\right)^{2} R_{1} R_{2} R_{3}} .
\end{aligned}
$$

This completes the general black ring solution as written in canonical coordinates (2.10)(2.11). Note that using (H.17) in Appendix [H] it is easy to see that $G_{33}$ can be written in the alternative form

$$
G_{33}=\frac{\left(R_{3}+z-\kappa^{2}\right)\left(R_{2}-z+c \kappa^{2}\right)}{R_{1}-z-c \kappa^{2}} .
$$




\section{Rod-structure}

We now analyze the rod-structure of the general black ring metric. This includes an analysis of the possible conical singularities of the solution. The rod-structure is as follows:

- The semi-infinite space-like $\operatorname{rod}\left[-\infty,-c \kappa^{2}\right]$. For $r=0$ and $z \in\left[-\infty,-c \kappa^{2}\right]$ we have that $R_{1}-R_{3}+(1+c) \kappa^{2}=0$ which using (6.7) is seen to give that $G_{33}=0$. This means we have a rod $\left[-\infty,-c \kappa^{2}\right]$ in the direction $v=(0,0,1)$, i.e. in the $\partial / \partial x^{3}$ direction. Using (3.10) we see furthermore that $x^{3}=\psi$ needs to have period

$$
\Delta \psi=2 \pi \frac{\sqrt{1-b}}{1-c},
$$

to avoid a conical singularity for $r=0$ and $z \in\left[-\infty,-c \kappa^{2}\right]$. Since $u=-1$ is equivalent to $R_{1}-R_{3}+(1+c) \kappa^{2}=0$ we see that this conical singularity corresponds to the one at $u=-1$ mentioned above.

- The finite time-like rod $\left[-c \kappa^{2}, c \kappa^{2}\right]$. For $r=0$ and $z \in\left[-c \kappa^{2}, c \kappa^{2}\right]$ we see that $R_{1}+R_{2}-2 c \kappa^{2}=0$. One can then check that $\sum_{j=1}^{3} G_{i j} v^{j}=0$ for $r=0$ and $z \in\left[-c \kappa^{2}, c \kappa^{2}\right]$ with $v$ being the vector

$$
v=(1, \Omega, 0), \quad \Omega=\frac{b-c}{(1-c) C \kappa} .
$$

From this we see that we have a rod $\left[-c \kappa^{2}, c \kappa^{2}\right]$ along the direction $v$ given in (6.13). The $\operatorname{rod}\left[-c \kappa^{2}, c \kappa^{2}\right]$ is time-like since $G_{i j} v^{i} v^{j} / r^{2}$ is negative for $r \rightarrow 0$. Note that $\Omega$ in (6.13) is the angular velocity of the general black ring solution. One can check that this rod corresponds to an event horizon with topology $S^{2} \times S^{1}$. This follows from the fact that the $\left[-c \kappa^{2}, c \kappa^{2}\right]$ rod has rods in the $\partial / \partial x^{3}$ direction on each side, so that the $z$ and $x^{3}$ coordinates parameterize the $S^{2}$ while the $x^{2}$ coordinate parameterize the $S^{1}$.

- The finite space-like $\operatorname{rod}\left[c \kappa^{2}, \kappa^{2}\right]$. For $r=0$ and $z \in\left[c \kappa^{2}, \kappa^{2}\right]$ we have that $R_{2}+$ $R_{3}-(1-c) \kappa^{2}=0$. Using (6.7) we see that this gives that $G_{33}=0$. This means we have a rod $\left[c \kappa^{2}, \kappa^{2}\right]$ in the direction $v=(0,0,1)$, i.e. in the $\partial / \partial x^{3}$ direction. Using (3.10) we see furthermore that $x^{3}=\psi$ needs to have period

$$
\Delta \psi=2 \pi \frac{\sqrt{1+b}}{1+c},
$$

to avoid a conical singularity for $r=0$ and $z \in\left[c \kappa^{2}, \kappa^{2}\right]$. However, since we have already fixed the period of $x^{3}=\psi$ by (6.12), curing the conical singularity associated with the rod $\left[c \kappa^{2}, \kappa^{2}\right]$ requires putting $b=2 c /\left(1+c^{2}\right)$. We do not fix $b$ in terms of $c$ here, thus we consider here solutions that can have conical singularities for $r=0$ and $z \in\left[c \kappa^{2}, \kappa^{2}\right]$. In Section 6.2 we consider the subset of solutions for which we do not have any conical singularities. Finally, note that since $u=1$ is equivalent to $R_{2}+R_{3}-(1-c) \kappa^{2}=0$ we see that this conical singularity corresponds to the one at $u=1$ mentioned above. 
- The semi-infinite space-like $\operatorname{rod}\left[\kappa^{2}, \infty\right]$. For $r=0$ and $z \in\left[\kappa^{2}, \infty\right]$ we have that $R_{1}-R_{3}-(1+c) \kappa^{2}=0$. Using (6.7) we see that this gives that $G_{12}=G_{22}=0$. This means we have a rod $\left[\kappa^{2}, \infty\right]$ in the direction $v=(0,1,0)$, i.e. in the $\partial / \partial x^{2}$ direction. Using (3.10) we see furthermore that $x^{2}=\phi$ needs to have period

$$
\Delta \phi=2 \pi \frac{\sqrt{1-b}}{1-c}
$$

to avoid a conical singularity for $r=0$ and $z \in\left[\kappa^{2}, \infty\right]$. Since $v=-1$ is equivalent to $R_{1}-R_{3}-(1+c) \kappa^{2}=0$ we see that this conical singularity corresponds to the one at $v=-1$ mentioned above.

\section{Static black ring}

We consider here briefly the case of the static black ring, obtained by setting $b=c$. The static black ring was first discussed in [16]. The static black ring is in the class of generalized Weyl solutions of [16] since its metric is diagonal.

Putting $b=c$ in (6.1) one easily gets the neutral black ring in C-metric coordinates. Note that $C=0, G(\xi)=\left(1-\xi^{2}\right) F(\xi), F(\xi)=1+c \xi$ and $0<c<1$. Using (6.7)-(6.10), we see that the static black ring metric in canonical coordinates (2.10)-(2.11) takes the form [16]

$$
\begin{gathered}
G_{11}=-\frac{R_{1}+R_{2}-2 c \kappa^{2}}{R_{1}+R_{2}+2 c \kappa^{2}}=-\frac{R_{1}-z-c \kappa^{2}}{R_{2}-z+c \kappa^{2}} \\
G_{22}=R_{3}-z+\kappa^{2}, G_{33}=\frac{\left(R_{3}+z-\kappa^{2}\right)\left(R_{2}-z+c \kappa^{2}\right)}{R_{1}-z-c \kappa^{2}} \\
e^{2 \nu}=\frac{\left(R_{1}+R_{2}+2 c \kappa^{2}\right)\left[(1-c) R_{1}+(1+c) R_{2}+2 c R_{3}\right]}{8\left(1-c^{2}\right) R_{1} R_{2} R_{3}}
\end{gathered}
$$

The static black ring metric have previously been written in canonical coordinates in [16 since it falls in the class of generalized Weyl solutions considered there. The rod-structure of the static black ring is:

- The semi-infinite space-like $\operatorname{rod}\left[-\infty,-c \kappa^{2}\right]$ in the $\partial / \partial x^{3}$ direction.

- The finite time-like $\operatorname{rod}\left[-c \kappa^{2}, c \kappa^{2}\right]$ in the $\partial / \partial x^{1}$ direction.

- The finite space-like rod $\left[c \kappa^{2}, \kappa^{2}\right]$ in the $\partial / \partial x^{3}$ direction.

- The semi-infinite space-like $\operatorname{rod}\left[\kappa^{2}, \infty\right]$ in the $\partial / \partial x^{2}$ direction.

We see that all the rods are rectangular relative to each other. The rod-structure of the static black ring was previously described in [16].

\section{Getting the Myers-Perry black hole from the general black ring solution}

We show here how one obtains the five-dimensional Myers-Perry rotating black hole solution with one angular momentum, that we considered in Section 5.3 from the general 
black ring solution. This has previously been described in Ref. 20] in terms of the C-metric coordinates used in the metric (6.1). Here we do it instead in terms of the canonical form of the metric (6.7)- (6.10).

We first note that we need to take the limit $c \rightarrow 1$, since the $\left[c \kappa^{2}, \kappa^{2}\right] \operatorname{rod}$ should be absent for the black hole solution. By considering explicit expressions for the mass $M$ and angular momentum $J_{1}$, plus the fact that $c \leq b<1$, one can see that $(1-b) /(1-c)$ and $\kappa^{2} /(1-c)$ should be fixed as $c \rightarrow 1$. One can furthermore see that we can find $\lambda$ and $\alpha$, so that

$$
c=1-\epsilon, \quad b=1-\epsilon \cos ^{2} \lambda, \quad \kappa=\frac{\sqrt{\alpha}}{\cos \lambda} \sqrt{\epsilon},
$$

with the limit being defined as $\epsilon \rightarrow 0$. Since $x^{2}$ and $x^{3}$ have periods (6.15) and (6.12) we need to make the rescaling

$$
x^{2}=\frac{\cos \lambda}{\sqrt{\epsilon}} \tilde{x}^{2}, \quad x^{3}=\frac{\cos \lambda}{\sqrt{\epsilon}} \tilde{x}^{3},
$$

so that now $\tilde{x}^{2}$ and $\tilde{x}^{3}$ have period $2 \pi$ for $\epsilon \rightarrow 0$. From the definition of the canonical $(r, z)$ coordinates, we see that this means we should make the rescaling

$$
r=\frac{\epsilon}{\cos ^{2} \lambda} \tilde{r}, \quad z=\frac{\epsilon}{\cos ^{2} \lambda} \tilde{z} .
$$

This gives that $\sqrt{r^{2}+\left(z \pm \kappa^{2}\right)^{2}}=\epsilon \sqrt{\tilde{r}^{2}+(\tilde{z} \pm \alpha)^{2}} / \cos ^{2} \lambda$. Using this with the metric (6.7)-(6.10) it is easy to see that one gets the metric (5.27) of a Myers-Perry rotating black hole with one angular momentum.

\subsection{Regular rotating black ring}

We now consider the regular black ring solution. In Section 6.1 we cured the conical singularities at the $\left[-\infty,-c \kappa^{2}\right]$ and $\left[\kappa^{2}, \infty\right]$ rods by imposing $x^{2}=\phi$ to have period (6.15) and $x^{3}=\psi$ to have period (6.12). However, we did not fix the potential conical singularity at the $\left[c \kappa^{2}, \kappa^{2}\right]$ rod. To ensure regularity at the $\left[c \kappa^{2}, \kappa^{2}\right] \operatorname{rod}, x^{3}=\psi$ should have period (6.14), which means we need to impose

$$
b=\frac{2 c}{1+c^{2}} .
$$

Therefore, with (6.20) imposed, and with $x^{2}=\phi$ and $x^{3}=\psi$ having their periods given by

$$
\Delta \phi=\Delta \psi=\frac{2 \pi}{\sqrt{1+c^{2}}},
$$

the rotating black ring solution (6.1) is regular [6, 20]. Note that the constant $C$ in (6.4) now takes the form

$$
C=\frac{2 c(1+c)}{1+c^{2}} \sqrt{\frac{1+c}{1-c}} .
$$


From (6.7) we get that the regular rotating black ring metric in canonical coordinates (2.10)-(2.11) is given by

$$
\begin{gathered}
G_{11}=-\frac{(1+c) R_{1}+(1-c) R_{2}-2 c R_{3}-4 c \kappa^{2}}{(1+c) R_{1}+(1-c) R_{2}-2 c R_{3}+4 c \kappa^{2}}, \\
G_{12}=-\frac{4 c \kappa \sqrt{1+c}}{\sqrt{1-c}} \frac{R_{3}-R_{1}+(1+c) \kappa^{2}}{(1+c) R_{1}+(1-c) R_{2}-2 c R_{3}+4 c \kappa^{2}}, \\
G_{33}=\frac{\left(R_{3}+z-\kappa^{2}\right)\left(R_{2}-z+c \kappa^{2}\right)}{R_{1}-z-c \kappa^{2}}, \\
e^{2 \nu}=\left[(1+c) R_{1}+(1-c) R_{2}-2 c R_{3}+4 c \kappa^{2}\right] \frac{(1-c) R_{1}+(1+c) R_{2}+2 c R_{3}}{8\left(1-c^{4}\right) R_{1} R_{2} R_{3}} .
\end{gathered}
$$

One can furthermore find $G_{22}$ using (6.9).

\section{Rod-structure}

The rod-structure of the regular rotating black ring solution is easily obtained from the rod-structure of the general black ring solution analyzed in Section 6.1 by imposing (6.20). We list here therefore only a short summary of the rod-structure of the regular rotating black ring:

- The semi-infinite space-like $\operatorname{rod}\left[-\infty,-c \kappa^{2}\right]$. This rod is in the direction $v=(0,0,1)$, i.e. in the $\partial / \partial x^{3}$ direction.

- The finite time-like rod $\left[-c \kappa^{2}, c \kappa^{2}\right]$. This rod is in the direction

$$
v=(1, \Omega, 0), \quad \Omega=\frac{1}{2 \kappa} \sqrt{\frac{1-c}{1+c}} .
$$

- The finite space-like $\operatorname{rod}\left[c \kappa^{2}, \kappa^{2}\right]$. This rod is in the direction $v=(0,0,1)$, i.e. in the $\partial / \partial x^{3}$ direction.

- The semi-infinite space-like $\operatorname{rod}\left[\kappa^{2}, \infty\right]$. This $\operatorname{rod}$ is in the direction $v=(0,1,0)$, i.e. in the $\partial / \partial x^{2}$ direction.

\section{Asymptotic region}

For the regular rotating black ring solution (6.23) in the asymptotic region $\sqrt{r^{2}+z^{2}} \rightarrow \infty$ with $z / \sqrt{r^{2}+z^{2}}$ finite, we find

$$
\begin{gathered}
G_{11}=-1+\frac{4 c \kappa^{2}}{1-c} \frac{1}{\sqrt{r^{2}+z^{2}}}+\mathcal{O}\left(\left(r^{2}+z^{2}\right)^{-1}\right), \\
G_{12}=-2 c \kappa^{3}\left(\frac{1+c}{1-c}\right)^{\frac{3}{2}} \frac{\sqrt{r^{2}+z^{2}}-z}{r^{2}+z^{2}}+\mathcal{O}\left(\left(r^{2}+z^{2}\right)^{-1}\right), \\
G_{22}=\left(\sqrt{r^{2}+z^{2}}-z\right)\left[1+\frac{\left(1+c+2 c^{2}\right) \kappa^{2}}{1-c} \frac{1}{\sqrt{r^{2}+z^{2}}}+\mathcal{O}\left(\left(r^{2}+z^{2}\right)^{-1}\right)\right], \\
G_{33}=\left(\sqrt{r^{2}+z^{2}}+z\right)\left[1+\frac{(2 c-1) \kappa^{2}}{\sqrt{r^{2}+z^{2}}}+\mathcal{O}\left(\left(r^{2}+z^{2}\right)^{-1}\right)\right] .
\end{gathered}
$$


Using (4.17) we then get

$$
M=\frac{3 \pi c \kappa^{2}}{(1-c)\left(1+c^{2}\right)}, \quad J_{1}=2 \pi c \kappa^{3}\left(\frac{1+c}{(1-c)\left(1+c^{2}\right)}\right)^{\frac{3}{2}}, \eta=\frac{3 \pi \kappa^{2}\left(1-c+2 c^{2}\right)}{2(1-c)\left(1+c^{2}\right)},
$$

along with $J_{2}=0$ and $\zeta=0$, where we used that $\varepsilon=1 / \sqrt{1+c^{2}}$ from (6.21). Note that

$$
\frac{J_{1}^{2}}{M^{3}}=\frac{4(1+c)^{3}}{27 \pi c} \text {. }
$$

We see that this has the minimum at $c=1 / 2$ with value $1 / \pi$. For $c \rightarrow 0$ it goes to infinity, while for $c=1$ it has the value $32 /(27 \pi)$. This is in accordance with [6, 22, 20].

\section{Discussion and conclusions}

The main results of this paper are as follows. We found in Section 2 that the metric of stationary and axisymmetric pure gravity solutions in $D$ dimensions can be written in the form (see Eqs. (2.10)-(2.11)

$$
d s^{2}=\sum_{i, j=1}^{D-2} G_{i j} d x^{i} d x^{j}+e^{2 \nu}\left(d r^{2}+d z^{2}\right), \quad r^{2}=|\operatorname{det} G|,
$$

apart from a subclass of solutions with constant $\operatorname{det} G$ that is considered in Appendix A The equation on the $D-2$ by $D-2$ dimensional symmetric matrix $G$ was found to take the simple form (see Eqs. (2.16))

$$
G^{-1} \vec{\nabla}^{2} G=\left(G^{-1} \vec{\nabla} G\right)^{2},
$$

where $\vec{\nabla}$ is the gradient on a three-dimensional flat Euclidean space, with metric (2.15). The function $\nu$ can then be found from $G$ using the integrable equations (2.13).

In Section 3 we considered then the behavior of $G$ for $r \rightarrow 0$. We generalized the concept of rods of [16] so that it can be used for stationary and axisymmetric solutions. One of the key points is that for each rod $\left[z_{1}, z_{2}\right]$ one has a direction in the $(D-2)$ dimensional vector space spanned by the Killing vector fields.

In Section 4 we analyzed the asymptotic region of four- and five-dimensional asymptotically flat solutions. In particular we identified how to read off the asymptotic quantities.

Finally, in Sections 5 and 6 we wrote down the metrics of the five-dimensional rotating black hole of Myers and Perry and the rotating black ring of Emparan and Reall in the canonical form (2.10)-(2.11). Furthermore, we analyzed the structure of the rods according to Section 3 and moreover the asymptotic region according to Section 4

The results of this paper have at least three interesting applications:

- Finding new stationary and axisymmetric solutions using the canonical form of the metric and the Einstein equations. For example one can look for new five-dimensional black ring solutions with two angular momenta, or for new solutions with a rotating black hole attached to a Kaluza-Klein bubble, as advocated in [8]. 
- Understanding the rod-structure of known stationary and axisymmetric solutions.

- Understanding better the uniqueness properties for higher-dimensional black holes. In four dimensions, the Carter-Robinson uniqueness theorem [3, 5] on the Kerr rotating black hole rests on using the Papapetrou form (1.1) of the metric. We expect therefore similar arguments to be applicable in higher dimensions, although they of course cannot prove any kind of strict uniqueness for five-dimensional rotating black holes due to the existence of rotating black rings.

\section{Acknowledgments}

We thank H. Elvang, N. Obers and P. Olesen for discussions and comments.

\section{A Special class of solutions}

In this appendix we consider stationary and axisymmetric solutions to the vacuum Einstein equations for which $\operatorname{det}\left(G_{i j}\right)$ is constant, with $G_{i j}$ defined by Eq. (2.4).

We can always find coordinates $(r, z)$ so that the metric (2.4) can be written

$$
d s^{2}=\sum_{i, j=1}^{D-2} G_{i j} d x^{i} d x^{j}+e^{2 \nu}\left(d r^{2}+d z^{2}\right) .
$$

This is possible since any two-dimensional manifold is conformally flat. The metric (A.1) is obviously the same as (2.10). However, the important difference is that the constraint (2.11) is replaced by restricting $\operatorname{det}\left(G_{i j}\right)$ to be constant.

Note first that demanding $\operatorname{det}\left(G_{i j}\right)$ to be constant leads to the identities

$$
\sum_{i, j=1}^{D-2} G^{i j} \partial_{a} G_{i j}=0, \quad \sum_{i, j=1}^{D-2} G^{i j} \partial_{a} \partial_{b} G_{i j}=\sum_{i, j, k, l=1}^{D-2} G^{i j} \partial_{a} G_{j k} G^{k l} \partial_{b} G_{l i},
$$

with $a, b=r, z$. Computing the Ricci tensor for the metric (A.1) and using the constraint that $\operatorname{det}\left(G_{i j}\right)$ is constant, we get that the vacuum Einstein equations can be written

$$
\begin{gathered}
\left(\partial_{r}^{2}+\partial_{z}^{2}\right) G_{i j}=\sum_{k, l=1}^{D-2} \partial_{r} G_{i k} G^{k l} \partial_{r} G_{l j}+\sum_{k, l=1}^{D-2} \partial_{z} G_{i k} G^{k l} \partial_{z} G_{l j} \\
\sum_{i, j, k, l=1}^{D-2} G^{i j} \partial_{r} G_{j k} G^{k l} \partial_{r} G_{l i}=\sum_{i, j, k, l=1}^{D-2} G^{i j} \partial_{z} G_{j k} G^{k l} \partial_{z} G_{l i} \\
\sum_{i, j, k, l=1}^{D-2} G^{i j} \partial_{r} G_{j k} G^{k l} \partial_{z} G_{l i}=0 \\
\left(\partial_{r}^{2}+\partial_{z}^{2}\right) \nu=-\frac{1}{8} \sum_{i, j, k, l=1}^{D-2}\left(G^{i j} \partial_{r} G_{j k} G^{k l} \partial_{r} G_{l i}+G^{i j} \partial_{z} G_{j k} G^{k l} \partial_{z} G_{l i}\right) .
\end{gathered}
$$

In conclusion, we can find solutions in the form of (A.1) with $\operatorname{det}\left(G_{i j}\right)$ being constant by first finding a $G_{i j}(r, z)$ solving (A.3) and then finding a solution for $\nu(r, z)$ of (A.4). 


\section{Four-dimensional examples}

In four dimensions, we have a well-known class of solutions to Eqs. A.3 - A.4 in the form of a particular kind of pp-wave solutions. These pp-wave solutions have

$$
G_{11}=-1-H(r, z), \quad G_{22}=1-H(r, z), \quad G_{12}=-H(r, z) .
$$

We see immediately that $\operatorname{det}\left(G_{i j}\right)=-1$. Furthermore, one can check that the Eqs. (A.3) with $D=4$ are solved, provided $H(r, z)$ obeys

$$
\left(\partial_{r}^{2}+\partial_{z}^{2}\right) H(r, z)=0
$$

Finally, $\nu(r, z)=0$ solves Eq. (A.4). Therefore, the pp-wave metrics

$$
d s^{2}=-d t^{2}+d x^{2}-H(d t+d x)^{2}+d r^{2}+d z^{2},
$$

with $H(r, z)$ obeying Eq. A.6), are in the class of solutions described by the metric (A.1) with $\operatorname{det}\left(G_{i j}\right)$ being constant [12]. Note moreover that any $\nu(r, z)$ solving $\left(\partial_{r}^{2}+\partial_{z}^{2}\right) \nu=0$ also gives a solution.

\section{B Analysis of $\operatorname{det}\left(G_{i j}\right)$}

In this appendix we study the behavior of $\operatorname{det}\left(G_{i j}\right)$ as a function. If we start with the metric (2.4) we can always put it in the form

$$
d s^{2}=\sum_{i, j=1}^{D-2} G_{i j} d x^{i} d x^{j}+C\left(d u^{2}+d v^{2}\right),
$$

where $C(u, v)$ and $G_{i j}(u, v)$ are functions only of $u$ and $v$. That we can bring the metric (2.4) to this form is easily seen from the fact that any two-dimensional manifold is conformally flat. Define now

$$
f=\sqrt{\left|\operatorname{det}\left(G_{i j}\right)\right|} .
$$

In the following we study the function $f(u, v)$. By computing the Ricci tensor for the metric (B.1) we get

$$
\sum_{i, j=1}^{D-2} G^{i j} R_{i j}=-\frac{1}{C f}\left(\frac{\partial^{2}}{\partial u^{2}}+\frac{\partial^{2}}{\partial v^{2}}\right) f .
$$

Now, since we consider solutions that are Ricci flat, we get that

$$
\left(\frac{\partial^{2}}{\partial u^{2}}+\frac{\partial^{2}}{\partial v^{2}}\right) f=0
$$

If we define the complex variable $\omega=u+i v$, along with the derivatives $\partial=\frac{\partial}{\partial u}+i \frac{\partial}{\partial v}$ and $\bar{\partial}=\frac{\partial}{\partial u}-i \frac{\partial}{\partial v}$, we see that $\bar{\partial} \partial f=0$. Therefore, $\partial f$ is a holomorphic function. We know from elementary complex analysis (see for example [23]) that either the zeroes of a holomorphic function are isolated or the function is identically zero (assuming the set that the function is defined on is simply connected). Since $\partial f=\frac{\partial f}{\partial u}+i \frac{\partial f}{\partial v}$ we can draw the conclusion: 
- Either $f(u, v)$ is a constant function or $\left(\frac{\partial f}{\partial u}, \frac{\partial f}{\partial v}\right) \neq(0,0)$ except in isolated points.

\section{Diagonalizing a two-dimensional metric}

In this appendix we prove, for the sake of clarity and completeness, the rather basic result that given a well-behaved function on a two-dimensional Riemannian manifold one can diagonalize the metric with the given function being one of the coordinates.

Consider a two-dimensional Riemannian manifold $M$ with a coordinate system $\left(y^{1}, y^{2}\right)$. Write the metric as

$$
d s^{2}=\hat{g}_{a b} d y^{a} d y^{b} \text {. }
$$

Let $z^{1}\left(y^{1}, y^{2}\right)$ be a given function with $\left(\frac{\partial z^{1}}{\partial y^{1}}, \frac{\partial z^{1}}{\partial y^{2}}\right) \neq(0,0)$. We want to show that we can find a function $z^{2}\left(y^{1}, y^{2}\right)$ so that $\left(z^{1}, z^{2}\right)$ is a new coordinate system and so that the metric in $z^{a}$ coordinates is diagonal, i.e. so that $g_{12}=0$, where we write the metric as $d s^{2}=g_{a b} d z^{a} d z^{b}$. Equivalently, we can demand that $g^{12}=0$. This is the same as

$$
\hat{g}^{a b} \frac{\partial z^{1}}{\partial y^{a}} \frac{\partial z^{2}}{\partial y^{b}}=0 \text {. }
$$

Now, define the vector field $V=V^{1} \frac{\partial}{\partial y^{1}}+V^{2} \frac{\partial}{\partial y^{2}}$ by

$$
V^{a}=\hat{g}^{a b} \frac{\partial z^{1}}{\partial y^{b}}
$$

Consider now the integral curves of $V$. Define an equivalence relation $\sim$ on $M$ where two points $p, q \in M$ are equivalent, i.e. $p \sim q$, if they are connected by an integral curve. Then we can define the quotient space $M / \sim$. Clearly, $M / \sim$ is a one-dimensional space. Let now $z^{2}$ be a coordinate on $M / \sim$. We then extend the scalar field $z^{2}$ on $M / \sim$ to a scalar field $z^{2}$ on $M$. Clearly, this scalar field $z^{2}$ on $M$ has the property that $z^{2}$ is constant on the integral curves of $V$. Since $z^{2}$ is constant on the integral curves of $V$ we get that

$$
V^{a} \frac{\partial z^{2}}{\partial y^{a}}=0
$$

which is the same as (C.2). We have therefore proven that for any given function $z^{1}\left(y^{1}, y^{2}\right)$ with $\left(\frac{\partial z^{1}}{\partial y^{1}}, \frac{\partial z^{1}}{\partial y^{2}}\right) \neq(0,0)$ we can find a function $z^{2}\left(y^{1}, y^{2}\right)$ such that

$$
d s^{2}=\hat{g}_{a b} d y^{a} d y^{b}=A\left(d z^{1}\right)^{2}+B\left(d z^{2}\right)^{2},
$$

and so that $\left(z^{1}, z^{2}\right)$ is a coordinate system on the two-dimensional manifold.

\section{Computation of Ricci tensor}

\section{Computation of the Ricci tensor with general $\Lambda$}

We consider first the $D$-dimensional metric

$$
d s^{2}=\sum_{i, j=1}^{D-2} G_{i j} d x^{i} d x^{j}+e^{2 \nu}\left(d r^{2}+\Lambda d z^{2}\right),
$$


with

$$
r=\sqrt{\left|\operatorname{det}\left(G_{i j}\right)\right|}
$$

where $G_{i j}, \nu$ and $\Lambda$ are functions of $r$ and $z$ only. The non-zero components of the Christoffel symbols for the metric (D.1) are

$$
\begin{gathered}
\Gamma_{i j}^{r}=-\frac{1}{2} e^{-2 \nu} \partial_{r} G_{i j}, \quad \Gamma_{i j}^{z}=-\frac{1}{2} e^{-2 \nu} \Lambda^{-1} \partial_{z} G_{i j}, \\
\Gamma_{r j}^{i}=\frac{1}{2} \sum_{k=1}^{D-2} G^{i k} \partial_{r} G_{j k}, \quad \Gamma_{z j}^{i}=\frac{1}{2} \sum_{k=1}^{D-2} G^{i k} \partial_{z} G_{j k}, \\
\Gamma_{r r}^{r}=\partial_{r} \nu, \quad \Gamma_{z z}^{z}=\partial_{z} \nu+\frac{1}{2 \Lambda} \partial_{z} \Lambda, \quad \Gamma_{r z}^{r}=\partial_{z} \nu, \quad \Gamma_{z r}^{z}=\partial_{r} \nu+\frac{1}{2 \Lambda} \partial_{r} \Lambda, \\
\Gamma_{z z}^{r}=-\Lambda \partial_{r} \nu-\frac{1}{2 C} \partial_{r} \Lambda, \quad \Gamma_{r r}^{z}=-\frac{1}{\Lambda} \partial_{z} \nu .
\end{gathered}
$$

Note now that since $r=\sqrt{\left|\operatorname{det} G_{i j}\right|}$ we have

$$
\sum_{i, j=1}^{D-2} G^{i j} \partial_{r} G_{i j}=\frac{2}{r}, \sum_{i, j=1}^{D-2} G^{i j} \partial_{z} G_{i j}=0
$$

Using this we get

$$
\sum_{i, j=1}^{D-2} G^{i j} \Gamma_{i j}^{r}=-\frac{1}{r} e^{-2 \nu}, \sum_{i, j=1}^{D-2} G^{i j} \Gamma_{i j}^{z}=0, \quad \sum_{i=1}^{D-2} \Gamma_{r i}^{i}=\frac{1}{r}, \quad \sum_{i=1}^{D-2} \Gamma_{z i}^{i}=0 .
$$

We compute then

$$
\begin{aligned}
2 e^{2 \nu} R_{i j}= & -\partial_{r}^{2} G_{i j}-\frac{1}{r} \partial_{r} G_{i j}-\frac{\partial_{r} \Lambda}{2 \Lambda} \partial_{r} G_{i j}-\frac{1}{\Lambda} \partial_{z}^{2} G_{i j}+\frac{\partial_{z} \Lambda}{2 \Lambda^{2}} \partial_{z} G_{i j} \\
& +\sum_{k, l=1}^{D-2} G^{k l} \partial_{r} G_{k i} \partial_{r} G_{l j}+\frac{1}{\Lambda} \sum_{k, l=1}^{D-2} G^{k l} \partial_{z} G_{k i} \partial_{z} G_{l j} .
\end{aligned}
$$

Notice now that from the fact that $r=\sqrt{\left|\operatorname{det} G_{i j}\right|}$ we have

$$
\begin{aligned}
& -\sum_{i, j=1}^{D-2} G^{i j} \partial_{r}^{2} G_{i j}+\sum_{i, j, k, l=1}^{D-2} G^{i j} G^{k l} \partial_{r} G_{k i} \partial_{r} G_{l j}=\frac{2}{r^{2}} \\
& -\sum_{i, j=1}^{D-2} G^{i j} \partial_{z}^{2} G_{i j}+\sum_{i, j, k, l=1}^{D-2} G^{i j} G^{k l} \partial_{z} G_{k i} \partial_{z} G_{l j}=0 .
\end{aligned}
$$

Using (D.7) together with (D.6), we get

$$
\sum_{i, j=1}^{D-2} G^{i j} R_{i j}=-\frac{\partial_{r} \Lambda}{2 e^{2 \nu} \Lambda r}
$$




\section{Computation of the Ricci tensor with $\Lambda=1$}

We now set $\Lambda=1$ in the metric (D.1). The non-zero components of the Ricci tensor can then be computed to be

$$
\begin{gathered}
2 e^{2 \nu} R_{i j}=-\left(\partial_{r}^{2}+\frac{1}{r} \partial_{r}+\partial_{z}^{2}\right) G_{i j}+\sum_{k, l=1}^{D-2} G^{k l} \partial_{r} G_{k i} \partial_{r} G_{l j}+\sum_{k, l=1}^{D-2} G^{k l} \partial_{z} G_{k i} \partial_{z} G_{l j} \\
R_{r r}=-\partial_{r}^{2} \nu-\partial_{z}^{2} \nu+\frac{1}{r^{2}}+\frac{1}{r} \partial_{r} \nu-\frac{1}{4} \sum_{i, j, k, l=1}^{D-2} G^{i j} G^{k l} \partial_{r} G_{i k} \partial_{r} G_{j l} \\
R_{z z}=-\partial_{r}^{2} \nu-\partial_{z}^{2} \nu-\frac{1}{r} \partial_{r} \nu-\frac{1}{4} \sum_{i, j, k, l=1}^{D-2} G^{i j} G^{k l} \partial_{z} G_{i k} \partial_{z} G_{j l} \\
R_{r z}=\frac{1}{r} \partial_{z} \nu-\frac{1}{4} \sum_{i, j, k, l=1}^{D-2} G^{i j} G^{k l} \partial_{r} G_{i k} \partial_{z} G_{j l} .
\end{gathered}
$$

\section{E Properties of the equations for $G_{i j}(r, z)$}

In this appendix we derive several useful properties of the equations (2.12) for $G_{i j}(r, z)$. We use in the following the formal rewriting of these equations in the form of Eq. (2.16).

Let $A(r, z)$ and $B(r, z)$ be $D-2$ times $D-2$ matrices obeying

$$
\left[A(r, z), B\left(r^{\prime}, z^{\prime}\right)\right]=0
$$

for any $(r, z)$ and $\left(r^{\prime}, z^{\prime}\right)$. Note that this means that $A(r, z)$ or any derivative of $A(r, z)$ commutes with $B(r, z)$ or any derivative of $B(r, z)$. Write now $G=A B$. Then we have

$$
G^{-1} \vec{\nabla}^{2} G-\left(G^{-1} \vec{\nabla} G\right)^{2}=A^{-1} \vec{\nabla}^{2} A-\left(A^{-1} \vec{\nabla} A\right)^{2}+B^{-1} \vec{\nabla}^{2} B-\left(B^{-1} \vec{\nabla} B\right)^{2} .
$$

From this equation we get the following lemma:

Lemma E.1 Let $A(r, z)$ and $B(r, z)$ be $D-2$ times $D-2$ matrices that commutes as in (E.1). If $A$ and $B$ obey the differential equations

$$
A^{-1} \vec{\nabla}^{2} A=\left(A^{-1} \vec{\nabla} A\right)^{2}, \quad B^{-1} \vec{\nabla}^{2} B=\left(B^{-1} \vec{\nabla} B\right)^{2},
$$

then the matrix $G=A B$ obeys $G^{-1} \vec{\nabla}^{2} G=\left(G^{-1} \vec{\nabla} G\right)^{2}$.

The consequence of this lemma is that we can combine solutions into new solutions, as long as (E.1) is obeyed. An important use of lemma E.1 is the following corollary:

Corollary E.2 Let $A(r, z)$ be a $D-2$ times $D-2$ matrix and let $f(r, z)$ be a function. If $G$ and $f$ obey the differential equations

$$
A^{-1} \vec{\nabla}^{2} A=\left(A^{-1} \vec{\nabla} A\right)^{2}, \quad \vec{\nabla}^{2} f=0,
$$

then the matrix $G=e^{f} A$ obeys $G^{-1} \vec{\nabla}^{2} G=\left(G^{-1} \vec{\nabla} G\right)^{2}$. 
Another important situation where the lemma E.1 can be applied and where the implication of lemma E.1 in fact can be reversed is expressed in the following lemma:

Lemma E.3 Let the $D-2$ times $D-2$ matrix $G(r, z)$ be such that $G=A \oplus B$, where $A(r, z)$ is a $k$ times $k$ matrix and $B(r, z)$ is a $D-2-k$ times $D-2-k$ matrix. I.e. $G$ is the geometric direct sum of $A$ and $B$. In this case, it is clear that $A$ and $B$ obey the differential equations

$$
A^{-1} \vec{\nabla}^{2} A=\left(A^{-1} \vec{\nabla} A\right)^{2}, \quad B^{-1} \vec{\nabla}^{2} B=\left(B^{-1} \vec{\nabla} B\right)^{2},
$$

if and only if $G$ obeys $G^{-1} \vec{\nabla}^{2} G=\left(G^{-1} \vec{\nabla} G\right)^{2}$.

This lemma can of course be used successively for the cases where $G(r, z)$ can be split up to the direct sum of several matrices acting on linearly independent subspaces, i.e. $G=A_{1} \oplus A_{2} \oplus \cdots \oplus A_{n}$. An important special case of this is when $G$ is diagonal. We have the following corollary of lemma E.3.

Corollary E.4 Let the $D-2$ times $D-2$ matrix $G(r, z)$ be a diagonal matrix

$$
G=\operatorname{diag}\left( \pm \exp \left(2 U_{1}\right), \exp \left(2 U_{2}\right), \ldots, \exp \left(2 U_{D-2}\right)\right)
$$

where $U_{i}(r, z), i=1, \ldots, D-2$, are functions. Then

$$
\vec{\nabla}^{2} U_{i}=0, \quad i=1, \ldots, D-2,
$$

if and only if $G^{-1} \vec{\nabla}^{2} G=\left(G^{-1} \vec{\nabla} G\right)^{2}$.

When $G(r, z)$ is diagonal it corresponds to a generalized Weyl solution (1.4) (see [16]), since the $D-2$ Killing vector fields are orthogonal. We see that (2.12) correctly reduce to (1.5). Moreover, it is clear that $\operatorname{det}\left(G_{i j}\right)= \pm r^{2}$ is equivalent to $\sum_{i=1}^{D-2} U_{i}=\log r$.

We have also a general result for the inverse of a matrix:

Lemma E.5 An $n$ by $n$ invertible matrix $G(r, z)$ obeys the equation $G^{-1} \vec{\nabla}^{2} G=\left(G^{-1} \vec{\nabla} G\right)^{2}$ if and only if the inverse matrix $G^{-1}$ obeys the corresponding equation

$$
G \vec{\nabla}^{2} G^{-1}=\left(G \vec{\nabla} G^{-1}\right)^{2}
$$

This lemma can be used to find new solutions from already known solutions. Of course, one has to remember that the complete $G$ matrix moreover should have $|\operatorname{det} G|=r^{2}$. The following corollary is one way to take this into account:

Corollary E.6 Let $G(r, z)$ be a $D-2$ by $D-2$ matrix with $|\operatorname{det} G|=r^{2}$ and $G^{-1} \vec{\nabla}^{2} G=$ $\left(G^{-1} \vec{\nabla} G\right)^{2}$. Then the matrix $M=r^{\frac{4}{D-2}} G^{-1}$ obeys $\operatorname{det} M=\operatorname{det} G$ and $M^{-1} \vec{\nabla}^{2} M=$ $\left(M^{-1} \vec{\nabla} M\right)^{2}$. 
We note that we can multiply constant matrices on solutions:

Lemma E.7 Let the $D-2$ by $D-2$ matrix $G(r, z)$ solve the equation $G^{-1} \vec{\nabla}^{2} G=$ $\left(G^{-1} \vec{\nabla} G\right)^{2}$ and let $A$ and $B$ be constant invertible matrices. Then the matrix $M=A G B$ obeys $M^{-1} \vec{\nabla}^{2} M=\left(M^{-1} \vec{\nabla} M\right)^{2}$.

Finally, an important and useful theorem that concerns systems with an orthogonal Killing vector is the following:

Theorem E.8 Consider the class of metrics with $G_{1 i}=0, i=2, \ldots, D-2$, i.e. with the Killing vector $V_{(1)}=\frac{\partial}{\partial x^{1}}$ being orthogonal to the $D-3$ other Killing vector fields. Then we can always write $G$ as

$$
G=s e^{2 U} \oplus e^{-\frac{2}{D-3} U} M=\left(\begin{array}{cccc}
s e^{2 U} & 0 & \cdots & 0 \\
0 & & \\
\vdots & e^{-\frac{2}{D-3} U} M \\
0 & &
\end{array}\right)
$$

where $s= \pm 1, U(r, z)$ is a function and $M(r, z)$ is a $D-3$ by $D-3$ symmetric real matrix with $\operatorname{det} M=s \operatorname{det} G$ so that $|\operatorname{det} M|=r^{2}$. Moreover, $G$ obeys $G^{-1} \vec{\nabla}^{2} G=\left(G^{-1} \vec{\nabla} G\right)^{2}$ if and only if

$$
\vec{\nabla}^{2} U=0, \quad M^{-1} \vec{\nabla}^{2} M=\left(M^{-1} \vec{\nabla} M\right)^{2} .
$$

Proof: It is trivial to see that we can always write $G$ on the form (E.9). That $G^{-1} \vec{\nabla}^{2} G=$ $\left(G^{-1} \vec{\nabla} G\right)^{2}$ if and only (E.10) is true follows from using lemma E.3 together with lemma E.2,

Theorem E.8 is useful since it allows one to take a $D=n$ dimensional solution and creating new non-trivial $D=n+1$ dimensional solutions. Moreover, for a $D=n$ dimensional solution with a Killing vector orthogonal to all other $D-3$ Killing vector fields we can reduce the system of equations to be a 3 -dimensional Laplace equation together with, but decoupled from, the equations for a $D=n-1$ dimensional solution.

\section{F Singularities at $r=0$}

We consider in this appendix what happens for solutions that have more than one eigenvalue of $G(r, z)$ going to zero for $r \rightarrow 0$. We restrict for simplicity here to the case with two eigenvalues going to zero for $r \rightarrow 0$. One can easily extend the argument to consider more than two eigenvalues.

We begin by considering the solution

$$
G_{11}=r^{2 a}, G_{22}=r^{2-2 a}, e^{2 \nu}=r^{-2 a(1-a)},
$$

where $0 \leq a \leq 1$. This solves the equations (2.12)-(2.13). We compute the curvature invariant

$$
R_{\mu \nu \rho \sigma} R^{\mu \nu \rho \sigma}=16 a^{2}(1-a)^{2}\left(1-a+a^{2}\right) r^{-4\left(1-a+a^{2}\right)}
$$


Since $1-a+a^{2}$ is strictly positive we see that there is a curvature singularity at $r=0$, unless $a=0$ or $a=1$ which in both cases corresponds to only having one eigenvalue going to zero. Therefore, for this solution we see that having two eigenvalues going to zero invariably leads to a curvature singularity.

Consider now a general solution for which we have an interval $\left[z_{1}, z_{2}\right]$ so that for any $z \in\left[z_{1}, z_{2}\right]$ we have that two eigenvalues of $G(r, z)$ going to zero for $r \rightarrow 0$. Then using the same type of arguments as in Section 3.1 we can make a constant orthogonal transformation of $G(r, z)$ so that $G_{1 i}(0, z)=G_{2 i}(0, z)=0$ for $i=1,2, \ldots, D-2$, for a given $z_{1}<z<z_{2}$. Therefore, given the structure of the equations (2.12)-(2.13), we see that we effectively can reduce this system to the example given by Eq. (F.1). In conclusion, we have shown that for any solution having two eigenvalues of $G(r, z)$ that go to zero as $r \rightarrow 0$ for a given $z$, we get curvature singularities, except perhaps in isolated points on the $z$-axis corresponding to the endpoints of the interval given above. As mentioned above, one can easily extend these arguments to consider more than two eigenvalues going to zero.

\section{G Prolate spherical coordinates}

We define here the prolate spherical coordinates $(x, y)$ which for certain stationary and axisymmetric solutions are convinient to use. The prolate spherical coordinates were introduced for four-dimensional stationary and axisymmetric solutions in 24] (see also [12, 14, 15]). The prolate spherical coordinates are used to describe rotating black hole solutions in Section 5 .

The prolate spherical coordinates $(x, y)$ are defined in terms of the canonical $(r, z)$ coordinates by

$$
r=\alpha \sqrt{\left(x^{2}-1\right)\left(1-y^{2}\right)}, \quad z=\alpha x y,
$$

where $\alpha>0$ is a constant. We take $x$ and $y$ to have the ranges

$$
x \geq 1, \quad-1 \leq y \leq 1
$$

We have

$$
d r^{2}+d z^{2}=\alpha^{2}\left(x^{2}-y^{2}\right)\left[\frac{d x^{2}}{x^{2}-1}+\frac{d y^{2}}{1-y^{2}}\right] .
$$

Note that Eqs. (2.12) can be written in prolate spherical coordinates as

$$
\partial_{x}\left[\left(x^{2}-1\right) \partial_{x} G\right]+\partial_{y}\left[\left(1-y^{2}\right) \partial_{y} G\right]=\left(x^{2}-1\right)\left(\partial_{x} G\right) G^{-1} \partial_{x} G+\left(1-y^{2}\right)\left(\partial_{y} G\right) G^{-1} \partial_{y} G
$$

We now give the transformation from $(x, y)$ coordinates to $(r, z)$ coordinates. Defining

$$
R_{+}=\sqrt{r^{2}+(z+\alpha)^{2}}, \quad R_{-}=\sqrt{r^{2}+(z-\alpha)^{2}},
$$

one can easily check using (G.1) that

$$
R_{+}=\alpha(x+y), \quad R_{-}=\alpha(x-y) .
$$


Therefore, we see that

$$
x=\frac{R_{+}+R_{-}}{2 \alpha}, y=\frac{R_{+}-R_{-}}{2 \alpha} .
$$

Furthermore, we note that

$$
R_{ \pm}+z \pm \alpha=\alpha(x \pm 1)(1+y), \quad R_{ \pm}-(z \pm \alpha)=\alpha(x \mp 1)(1-y) .
$$

If we consider the asymptotic region $\sqrt{r^{2}+z^{2}} \rightarrow \infty$ with $z / \sqrt{r^{2}+z^{2}}$ finite, we see that

$$
x \simeq \frac{1}{\alpha} \sqrt{r^{2}+z^{2}}, \quad y \simeq \frac{z}{\sqrt{r^{2}+z^{2}}} .
$$

Thus, the asymptotic region in terms of the prolate spherical coordinates is $x \rightarrow \infty$ and $y$ being finite.

\section{H C-metric coordinates}

We consider in this section the coordinate transformation from general C-metric coordinates to the canonical $(r, z)$ coordinates. ${ }^{13}$ The general C-metric coordinates $(u, v)$ are here defined in relation to $(r, z)$ by

$$
\begin{gathered}
r=\frac{2 \kappa^{2} \sqrt{-G(u) G(v)}}{(u-v)^{2}}, \\
e^{2 \nu}\left(d r^{2}+d z^{2}\right)=\zeta(u, v)\left(\frac{d u^{2}}{G(u)}-\frac{d v^{2}}{G(v)}\right),
\end{gathered}
$$

where $\kappa$ is a constant, and $\zeta(u, v)$ and $G(\xi)$ are functions that depend on the particular solution that we consider. The goal is now to find the $z$ coordinate. From $g^{r z}=0$ we find

$$
\frac{\partial z}{\partial v}=-\frac{g_{v v}}{g_{u u}} \frac{\partial r}{\partial u}\left(\frac{\partial r}{\partial v}\right)^{-1} \frac{\partial z}{\partial u} .
$$

Using this together with $g_{u v}=0$ one can easily derive that

$$
\frac{\partial z}{\partial u}=s \sqrt{\frac{g_{u u}}{g_{v v}}} \frac{\partial r}{\partial v}, \quad \frac{\partial z}{\partial v}=-s \sqrt{\frac{g_{v v}}{g_{u u}}} \frac{\partial r}{\partial u}, \quad s= \pm 1 .
$$

This gives

$$
s \frac{\partial z}{\partial u}=-\frac{\kappa^{2} G^{\prime}(v)}{(u-v)^{2}}-\frac{4 \kappa^{2} G(v)}{(u-v)^{3}}, \quad s \frac{\partial z}{\partial v}=-\frac{\kappa^{2} G^{\prime}(u)}{(u-v)^{2}}+\frac{4 \kappa^{2} G(u)}{(u-v)^{3}} .
$$

The integrability condition

$$
\frac{\partial}{\partial v} \frac{\partial z}{\partial u}=\frac{\partial}{\partial u} \frac{\partial z}{\partial v}
$$

is satisfied if and only if

$$
G^{\prime \prime}(v)+\frac{6 G^{\prime}(v)}{u-v}+\frac{12 G(v)}{(u-v)^{2}}=G^{\prime \prime}(u)-\frac{6 G^{\prime}(u)}{u-v}+\frac{12 G(u)}{(u-v)^{2}} .
$$

\footnotetext{
${ }^{13}$ See [25] for a review of the C-metric and the transformation from C-metric coordinates to canonical $(r, z)$ coordinates in the context of four-dimensional Weyl solutions.
} 
Integrating (․․․․ $)$, we get

$$
s z=b(v)+\frac{\kappa^{2} G^{\prime}(v)}{(u-v)}+\frac{2 \kappa^{2} G(v)}{(u-v)^{2}}=c(u)-\frac{\kappa^{2} G^{\prime}(u)}{(u-v)}+\frac{2 \kappa^{2} G(u)}{(u-v)^{2}},
$$

where $b(\xi)$ and $c(\xi)$ are two functions. Using the integrability condition (ㅍ.6) we see that

$$
\begin{aligned}
s\left(z-z_{0}\right) & =\frac{\kappa^{2} G^{\prime \prime}(v)}{6}+\frac{\kappa^{2} G^{\prime}(v)}{(u-v)}+\frac{2 \kappa^{2} G(v)}{(u-v)^{2}} \\
& =\frac{\kappa^{2} G^{\prime \prime}(u)}{6}-\frac{\kappa^{2} G^{\prime}(u)}{(u-v)}+\frac{2 \kappa^{2} G(u)}{(u-v)^{2}},
\end{aligned}
$$

where $z_{0}$ is a constant. Now, if we take $G(\xi)$ to be of the form

$$
G(\xi)=a_{0}+a_{1} \xi+a_{2} \xi^{2}+a_{3} \xi^{3}+a_{4} \xi^{4}
$$

one can check that (H.6) is obeyed.

Note that $e^{2 \nu}$ and $\zeta(u, v)$ in (H.1) are connected through the formula

$$
\frac{\zeta(u, v)}{e^{2 \nu}}=\frac{\kappa^{4}}{(u-v)^{4}}\left[G(u)\left(G^{\prime}(v)+\frac{4 G(v)}{u-v}\right)^{2}-G(v)\left(G^{\prime}(u)-\frac{4 G(u)}{u-v}\right)^{2}\right] .
$$

This formula is useful for finding $e^{2 \nu}$.

Consider now the particular choice of $G(\xi)$

$$
G(\xi)=\left(1-\xi^{2}\right)(1+c \xi),
$$

which correspond to the case of the five-dimensional black ring metric described in Section 6. If we take $s=1$ and $z_{0}=1 / 6$ we get

$$
z=\frac{\kappa^{2}(1-u v)(2+c u+c v)}{(u-v)^{2}} .
$$

We now look for constants $q$ and $\beta$ that solves the equation

$$
r^{2}+\left(z-q \kappa^{2}\right)^{2}=\frac{\kappa^{4}[\beta-c u v-2 q(u+v)]^{2}}{(u-v)^{2}} .
$$

There are precisely three solutions to this equation:

$$
q=-c, \beta=2+c ; \quad q=c, \beta=-2+c ; \quad q=1, \beta=-c .
$$

Write now

$$
R_{i}=\sqrt{r^{2}+\left(z-z_{i}\right)^{2}}, \quad z_{1}=-c \kappa^{2}, \quad z_{2}=c \kappa^{2}, \quad z_{3}=\kappa^{2}
$$

We get

$$
\begin{gathered}
R_{1}=\frac{\kappa^{2}[2+c(1+u+v-u v)]}{(u-v)}, \quad R_{2}=\frac{\kappa^{2}[2+c(-1+u+v+u v)]}{(u-v)}, \\
R_{3}=\frac{\kappa^{2}[-c(1+u v)-(u+v)]}{(u-v)} .
\end{gathered}
$$


We have furthermore that

$$
\begin{aligned}
& R_{1}+z-z_{1}=\frac{2 \kappa^{2}(1+u)(1-v)(1+c u)}{(u-v)^{2}}, \quad R_{1}-z+z_{1}=\frac{2 \kappa^{2}(1-u)(-1-v)(1+c v)}{(u-v)^{2}}, \\
& R_{2}+z-z_{2}=\frac{2 \kappa^{2}(1+u)(1-v)(1+c v)}{(u-v)^{2}}, \quad R_{2}-z+z_{2}=\frac{2 \kappa^{2}(1-u)(-1-v)(1+c u)}{(u-v)^{2}}, \\
& R_{3}+z-z_{3}=\frac{2 \kappa^{2}\left(1-u^{2}\right)(1+c v)}{(u-v)^{2}}, \quad R_{3}-z+z_{3}=\frac{2 \kappa^{2}\left(v^{2}-1\right)(1+c u)}{(u-v)^{2}} .
\end{aligned}
$$

Finally, we can solve for $u$ and $v$ to obtain

$$
\begin{aligned}
& u=\frac{(1-c) R_{1}-(1+c) R_{2}-2 R_{3}+2\left(1-c^{2}\right) \kappa^{2}}{(1-c) R_{1}+(1+c) R_{2}+2 c R_{3}}, \\
& v=\frac{(1-c) R_{1}-(1+c) R_{2}-2 R_{3}-2\left(1-c^{2}\right) \kappa^{2}}{(1-c) R_{1}+(1+c) R_{2}+2 c R_{3}} .
\end{aligned}
$$

\section{References}

[1] R. P. Kerr, "Gravitational field of a spinning mass as an example of algebraically special metrics," Phys. Rev. Lett. 11 (1963) 237-238.

[2] W. Israel, "Event horizons in static vacuum space-times," Phys. Rev. 164 (1967) 1776-1779.

[3] B. Carter, "Axisymmetric black hole has only two degrees of freedom," Phys. Rev. Lett. 26 (1971) 331-333.

[4] S. W. Hawking, "Black holes in General Relativity," Commun. Math. Phys. 25 (1972) 152-166.

[5] D. C. Robinson, "Uniqueness of the Kerr black hole," Phys. Rev. Lett. 34 (1975) 905-906.

[6] R. Emparan and H. S. Reall, "A rotating black ring in five dimensions," Phys. Rev. Lett. 88 (2002) 101101, hep-th/0110260

[7] R. C. Myers and M. J. Perry, "Black holes in higher dimensional space-times," Ann. Phys. 172 (1986) 304.

[8] H. Elvang, T. Harmark, and N. A. Obers, "Sequences of bubbles and holes: New phases of Kaluza-Klein black holes," hep-th/0407050

[9] H. Elvang, T. Harmark, and N. A. Obers, "Phases of Kaluza-Klein black holes," Class. Quant. Grav. 21 (2004) S1509-S1516.

[10] A. Papapetrou, "Eine rotationssymmetrische lösung in der allgemeinen relativitätstheorie," Ann. Physik. 12 (1953) 309-315. 
[11] A. Papapetrou, "Champs gravitationels stationaries a symmetrie axiale," Ann. Inst. H. Poincaré A 4 (1966) 83-105.

[12] H. Stephani, D. Kramer, M. Maccallum, C. Hoenselaers, and E. Herlt, Exact Solutions to Einstein's Field Equations: Second Edition. Cambridge University Press, 2003.

[13] R. M. Wald, General Relativity. The University of Chicago Press, 1984.

[14] S. Chandrasekhar, The Mathematical Theory of Black Holes. Oxford University Press, 1992.

[15] M. Heusler, Black Hole Uniqueness Theorems. Cambridge University Press, 1996.

[16] R. Emparan and H. S. Reall, "Generalized Weyl solutions," Phys. Rev. D65 (2002) 084025, hep-th/0110258.

[17] C. Charmousis and R. Gregory, "Axisymmetric metrics in arbitrary dimensions," Class. Quant. Grav. 21 (2004) 527-554, gr-qc/0306069.

[18] H. Weyl, "Zur gravitationstheorie," Ann. Phys. 54 (1917) 117.

[19] F. R. Tangherlini, "Schwarzschild field in $n$ dimensions and the dimensionality of space problem," Nuovo Cimento 27 (1963) 636.

[20] R. Emparan, "Rotating circular strings, and infinite non-uniqueness of black rings," JHEP 03 (2004) 064, hep-th/0402149.

[21] K. Hong and E. Teo, "A new form of the C-metric," Class. Quant. Grav. 20 (2003) 3269-3277, gr-qc/0305089.

[22] H. Elvang, "A charged rotating black ring," hep-th/0305247.

[23] F. J. Flanigan, Complex Variables: Harmonic and Analytic Functions. Dover Publications, 1972.

[24] D. M. Zipoy, "Topology of some spheroidal metrics," JMP 7 (1966) 1137.

[25] V. Pravda and A. Pravdova, "Boost-rotation symmetric spacetimes - review," Czech. J. Phys. 50 (2000) 333-376, gr-qc/0003067. 